\title{
Diastereoselective Synthesis of (-)-Bestatin, Epibestatin, Phebestin and (3S,4R)-4-Amino-3-hydroxy-5-phenylpentanoic Acid from an Aldehyde Derived from D-Phenylalanine
}

\author{
Vipin Kumar Jain* (D) \\ Department of Chemistry, Indian Institute of Technology \\ Kanpur, Kanpur-208016, India \\ jain91vipin@gmail.com
}

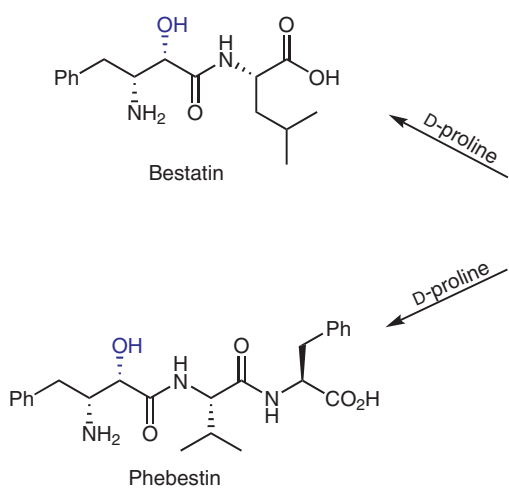

Phebestin

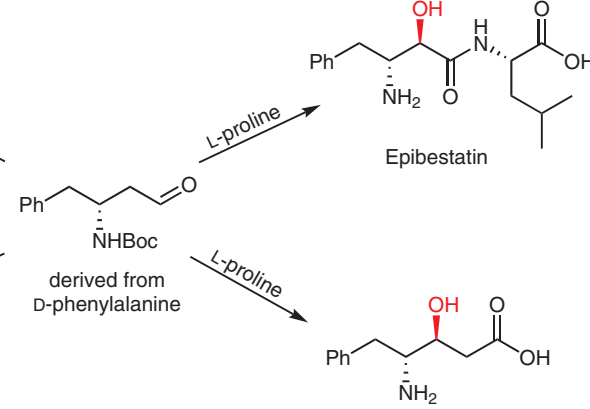

4-amino-3-hydroxy-5phenylpentanoic acid
Received: 27.09.2019

Accepted after revision: 09.10.2019

Published online: 25.10 .2019

DOI: 10.1055/s-0039-1690223; Art ID: so-2019-h0027-op

License terms: (c)

(c) 2019. The Author(s). This is an open access article published by Thieme under the terms of the Creative Commons Attribution-NonDerivative-NonCommercialLicense, permitting copying and reproduction so long as the original work is given appropriate credit. Contents may not be used for commercial purposes or adapted, remixed, transformed or built upon. (https://creativecommons.org/licenses/by-nc-nd/4.0/)

Abstract A convenient and efficient method for the synthesis of (-)bestatin, epibestatin, phebestin, and (3S,4R)-4-amino-3-hydroxy-5phenylpentanoic acid is reported. The key step is a proline-catalyzed $\alpha$ hydroxylation of an aldehyde derived from D-phenylalanine, which leads to incorporation of a hydroxyl group at the $\alpha$-position of that aldehyde with good yield and very high diastereoselectivity. Bestatin and its diastereomer epibestatin are synthesized from the same starting material using the same sequence of reactions, except for proline as the catalyst. An O-MOM and Boc-protected amino acid, a common intermediate for bestatin, was coupled with a dipeptide, $\mathrm{H}$-Val-Phe-OMe followed by global deprotection to yield phebestin. (3S,4R)-4-Amino-3-hydroxy-5phenylpentanoic acid was also synthesized in eight steps from the same starting material. The reported synthetic route offers a general method for the synthesis of such types of compounds and their analogues by changing the proline catalyst and/or the starting material from D- to Lphenylalanine.

Key words asymmetric hydroxylation, organocatalysis, reductive cleavage

(-)-Bestatin (Ubenimex) is a dipeptide containing an $\alpha$ hydroxy- $\beta$-amino amide subunit that was first isolated from Streptomyces olivoreticulithe by Umezawa et al. in 1976. ${ }^{1,2}$ It is an aminopeptidase inhibitor that exhibits immunostimulatory activity as well as cytotoxic activity. ${ }^{3,4}$ It is used clinically for the treatment of cancer, HIV, hypertension, and shows potential as an anti-inflammatory agent. ${ }^{5-8}$
Structure modification studies of bestatin and similar molecules such as phebestin, a tripeptide, indicate that biological activities of these molecules are significantly influenced by the (2S)-syn-stereochemistry of the hydroxyl group..$^{9,10}$

Various stereoselective methods for the synthesis of bestatin, phebestin ${ }^{11-27}$ and epibestatin ${ }^{28,29}$ are available and most of them utilized D-phenylalanine as a chiral starting material. Reported herein is an alternative and short method for the synthesis of bestatin, epibestatin, phebestin and (3S,4R)-4-amino-3-hydroxy-5-phenylpentanoic acid using proline-catalysed asymmetric $\alpha$-hydroxylation of an aldehyde derived from D-phenylalanine. The structures of these compounds are shown in Figure 1.

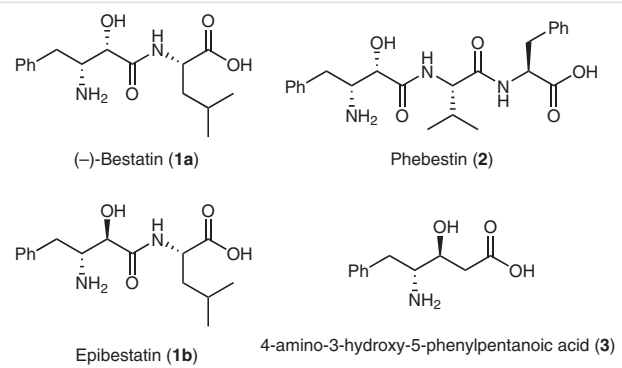

Figure 1

Proline-catalysed $\alpha$-hydroxylation of an aldehyde using nitrosobenzene followed by reduction of the $\mathrm{N}-\mathrm{O}$ bond is an attractive method to introduce a hydroxyl group stereoselectively. ${ }^{30-32}$ The aldehyde functional group can be further reduced to an alcohol or converted into an alkene through Wittig reaction in order to avoid racemization at the $\alpha$-position. As the part of our studies towards the synthesis of various bioactive and naturally occurring mole- 
cules, ${ }^{32-41}$ we recently reported the synthesis of D-threosphinganine, L-erythro-sphinganine and (-)-spisulosine from an aldehyde derived from aspartic acid. ${ }^{42}$

In the retrosynthetic analysis, it was anticipated that both bestatin and epibestatin could be synthesized from acid 9 using peptide coupling followed by deprotection of the Boc and MOM groups. Diol $\mathbf{5}$ could be obtained from aldehyde $\mathbf{4}$ using an $\alpha$-hydroxylation reaction. Compound $\mathbf{9 a}$ could be converted into phebestin. Olefin 15 could be obtained from aldehyde $\mathbf{4}$ using an $\alpha$-hydroxylation reaction followed by Wittig reaction and would yield compound $\mathbf{3}$ as shown in Scheme 1.

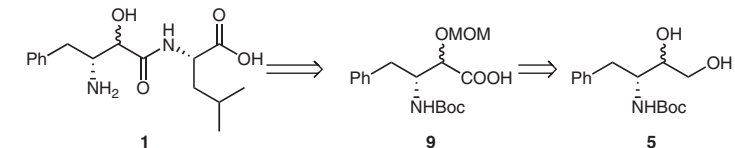

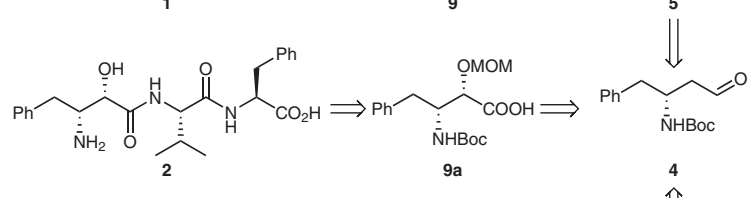

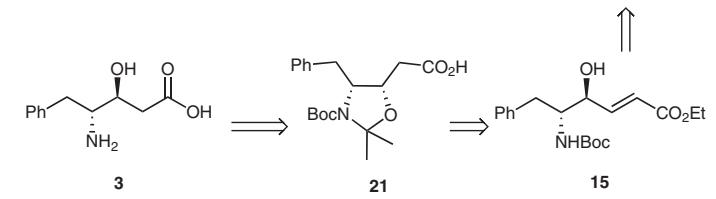

Scheme 1 Retrosynthetic analysis of compounds $\mathbf{1 , 2}$ and $\mathbf{3}$ from $\mathbf{4}$

Aldehyde 4 (for preparation see the literature ${ }^{43}$ ) was subjected to diastereoselective hydroxylation using nitrosobenzene, and D-proline as catalyst and subsequently reduced to the corresponding primary alcohol by $\mathrm{NaBH}_{4}$ in one pot. The crude product was further subjected to $\mathrm{N}-\mathrm{O}$ bond cleavage using $\mathrm{Cu}(\mathrm{OAc})_{2}$ to give diol $\mathbf{5 a}$ in $66 \%$ yield overall. It was observed by ${ }^{1} \mathrm{H}$ NMR spectroscopy that the hydroxylation reaction proceeded with 90:10 diastereoselectivity. The primary and secondary hydroxyl groups of compound $\mathbf{5 a}$ were protected as their TBDPS and MOM derivatives, respectively, to obtain the fully protected compound 7a in $64 \%$ overall yield. TBAF was then used to remove the silyl protecting group in compound $7 \mathbf{a}$ to furnish the primary alcohol 8a in $89 \%$ yield, which was then treated with PDC in DMF to produce the corresponding carboxylic acid $9 a$ in $76 \%$ yield (Scheme 2 ).

The fully protected $\alpha$-hydroxy- $\beta$-amino acid $\mathbf{9 a}$ is the precursor for the synthesis of both bestatin and phebestin. To obtained bestatin, compound 9a was coupled with the benzyl ester of L-leucine in the presence of EDC.HCl, HOBt and DIPEA to give the corresponding fully protected dipeptide 10a in $82 \%$ yield. Compound 10a was further subjected to Pd-catalysed hydrogenolysis followed by acidolysis of the Boc and MOM groups to furnish target molecule 1a from 10a in $86 \%$ yield (Scheme 2 ).

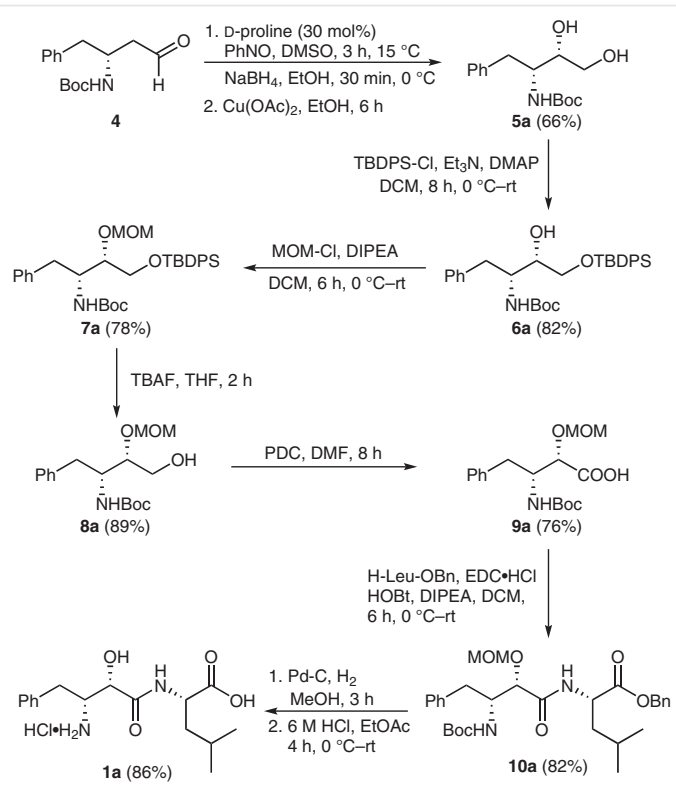

Scheme 2 Synthesis of bestatin (1a)

Epibestatin 1 $\mathbf{1 b}$ was obtained in an overall yield of $22 \%$ from aldehyde $\mathbf{4}$ using exactly the same sequence of reactions but using L-proline in the asymmetric $\alpha$-hydroxylation reaction (Scheme 3 ) leading to a diastereomer ratio of $87: 13$ as judged by ${ }^{1} \mathrm{H}$ NMR spectroscopy. Epibestatin is available in very limited quantities commercially and to date only a few synthetic strategies have been reported..$^{28,29}$
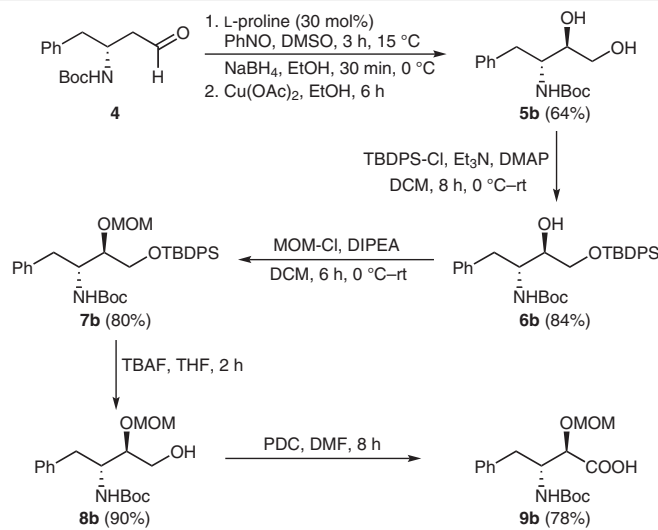

H-Leu-OBn, EDC.HCI $\mathrm{HOBt}$, DIPEA, DCM,
$6 \mathrm{~h}, 0^{\circ} \mathrm{C}-\mathrm{rt}$

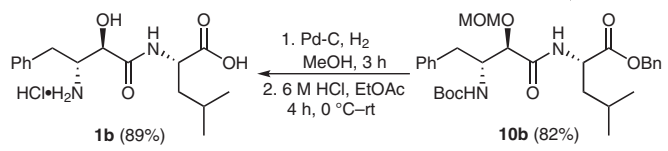

Scheme 3 Synthesis of epibestatin (1b)

To synthesize phebestin, compound 9a was coupled with dipeptide 12, which was obtained from coupling the methyl ester of L-phenylalanine with $\mathrm{NH}$-Boc protected 
L-valine, to give the fully protected tripeptide $\mathbf{1 3}$ in $70 \%$ yield. Hydrolysis of the methyl ester using LiOH followed by acidolysis of the Boc and MOM groups furnished the target molecule $\mathbf{2}$ in $89 \%$ yield over two steps (Scheme 4 ).

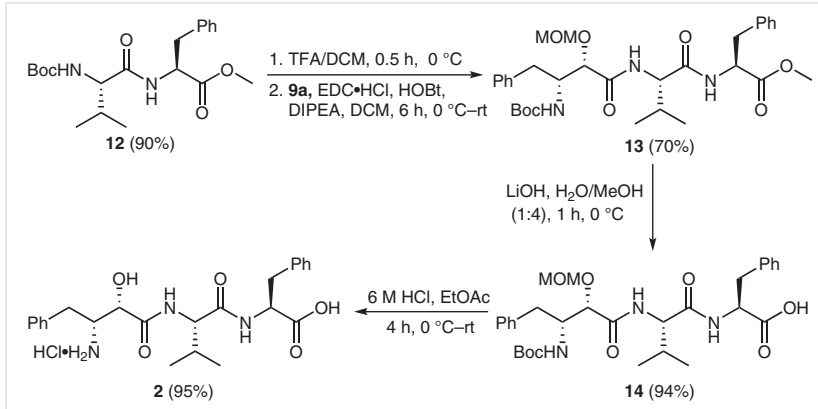

Scheme 4 Synthesis of phebestin (2)

$\beta$-Hydroxy- $\gamma$-amino acids have been designed for biologically active peptide mimics and for HIV protease inhibitors. Stictamide A, tasiamide B and hapolosin are biologically important compounds that contain 4-amino-3-hydroxy5-phenylpentanoic acid as a structural fragment. The activities of such compounds depend on the stereochemistries of both the amino- and hydroxyl groups. ${ }^{44,45} \mathrm{~A}$ variety of stereoselective methods for the synthesis of these acids and their analogues is available. ${ }^{46-50}(3 S, 4 R)$-4-Amino-3-hydroxy-5-phenylpentanoic acid (3) was also synthesized from the same starting material $\mathbf{4}$ in eight steps and in an overall yield of $15 \%$ (Scheme 5 ).

Thus, aldehyde $\mathbf{4}$ was subjected to L-proline-catalysed asymmetric $\alpha$-hydroxylation and subsequent Wittig reaction in one pot. The crude product was further treated with $\mathrm{Cu}(\mathrm{OAc})_{2}$ leading to cleavage of the $\mathrm{N}-\mathrm{O}$ bond to form olefin $\mathbf{1 5}$ in 70\% overall yield (Scheme 5 ).

Both the hydroxyl and amino groups in compound $\mathbf{1 5}$ were protected as an oxazolidine using 2,2-dimethoxypropane (DMP) and a catalytic amount of $p$-TsOH to $\mathbf{1 6}$ in $85 \%$ yield. $\mathrm{LiBH}_{4}$ was used to reduce compound $\mathbf{1 6}$ to primary alcohol 17 in $80 \%$ yield, and this was then oxidized to aldehyde 18 using 2-iodoxybenzoic acid (IBX) in $88 \%$ yield. The aldehyde 18 was subjected to L-proline-catalysed asymmetric $\alpha$-hydroxylation reaction followed by reduction and $\mathrm{N}-\mathrm{O}$ bond cleavage using $\mathrm{NaBH}_{4}$ and $\mathrm{Cu}(\mathrm{OAc})_{2}$, respectively, to furnish diol 19 in $65 \%$ overall yield. $\mathrm{NaIO}_{4}$ was used to cleave the diol to produce aldehyde $\mathbf{2 0}$, which was further oxidised to an acid 21 using PDC in 57\% yield after two steps. Acidolysis of the Boc group and oxazolidine ring in compound 21 furnished $\mathbf{3}$ in $98 \%$ yield (Scheme 5).

In conclusion, we have demonstrated a convenient and efficient route for the synthesis of bestatin, epibestatin, phebestin and $(3 S, 4 R)$-4-amino-3-hydroxy-5-phenylpentanoic acid using proline-catalysed $\alpha$-hydroxylation of an aldehyde derived from D-phenylalanine with high diastereo-
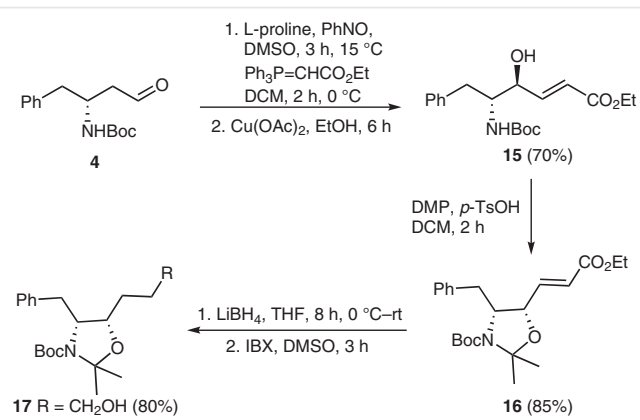

$\begin{aligned} 17 \mathrm{R} & =\mathrm{CH}_{2} \mathrm{OH}(80 \%) \\ 18 \mathrm{R} & =\mathrm{CHO}(88 \%)\end{aligned}$

1. L-proline (30 mol\%) $\mathrm{PhNO}, \mathrm{DMSO}, 3 \mathrm{~h}, 15^{\circ} \mathrm{C}$
$\mathrm{NaBH}_{4}, \mathrm{EtOH}, 30 \mathrm{~min}, 0^{\circ} \mathrm{C}$ 2. $\mathrm{Cu}(\mathrm{OAc})_{2}, \mathrm{EtOH}, 6 \mathrm{~h}$
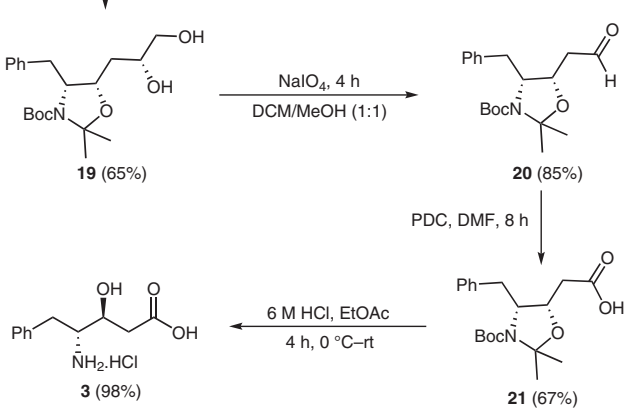

Scheme 5 Synthesis of $\mathbf{3}$ from aldehyde $\mathbf{4}$

selectivities and in good overall yields. The method described here offers a general method to synthesize several similar molecules using an organocatalytic route.

See the Supporting Information for general information.

Asymmetric $\alpha$-Hydroxylation of Aldehydes; General Procedure

To a stirred solution of aldehyde $4(1.00 \mathrm{~g}, 3.80 \mathrm{mmol})$ and nitrosobenzene $(0.44 \mathrm{~g}, 4.18 \mathrm{mmol})$ in anhydrous DMSO $(10 \mathrm{~mL})$, D- or L-proline $(0.13 \mathrm{~g}, 1.14 \mathrm{mmol}, 30 \mathrm{~mol} \%)$ was added at $15{ }^{\circ} \mathrm{C}$. The mixture was stirred for $3 \mathrm{~h}$ at the same temperature, then cooled to $0{ }^{\circ} \mathrm{C}$ and $\mathrm{NaBH}_{4}(0.28 \mathrm{~g}, 7.60 \mathrm{mmol})$ in EtOH $(15 \mathrm{~mL})$ was added and the mixture was stirred vigorously for $30 \mathrm{~min}$ at $0{ }^{\circ} \mathrm{C}$. On complete disappearance of starting material, the reaction was quenched with saturated aqueous $\mathrm{NH}_{4} \mathrm{Cl}(30 \mathrm{~mL})$ and the mixture was extracted with EtOAc (2 $\times 30 \mathrm{~mL}$ ). The combined organic phases were washed with brine (30 $\mathrm{mL}$ ), dried over $\mathrm{Na}_{2} \mathrm{SO}_{4}$, filtered, and concentrated. The crude aminohydroxylated product was taken as such to the next step leading to the cleavage of $\mathrm{O}-\mathrm{N}$ bond.

$\mathrm{Cu}(\mathrm{OAc})_{2}(0.17 \mathrm{~g}, 0.96 \mathrm{mmol})$ was added to a stirred solution of the above product in EtOH $(15 \mathrm{~mL})$ and the mixture was stirred vigorously for $6 \mathrm{~h}$ at room temperature. On complete disappearance of starting material, the reaction was quenched with saturated aqueous $\mathrm{NH}_{4} \mathrm{Cl}$ $(20 \mathrm{~mL})$ and extracted with EtOAc $(2 \times 20 \mathrm{~mL})$. The combined organic phases were washed with brine $(30 \mathrm{~mL})$, dried over $\mathrm{Na}_{2} \mathrm{SO}_{4}$, filtered, concentrated, and purified by column chromatography.

The same procedure was used for the preparation of compound 19. 


\section{tert-Butyl ((2R,3S)-3,4-Dihydroxy-1-phenylbutan-2-yl)carbamate} (5a)

Column chromatography (petroleum ether/EtOAc, 60:40).

Yield: $0.70 \mathrm{~g}$ (66\%); clear oil; $[\alpha]_{\mathrm{D}}{ }^{27}+18.97$ (c 1.22, $\mathrm{CHCl}_{3}$ ).

IR (thin film): 3382, 3063, 3028, 2924, 2854, 1682, $1604 \mathrm{~cm}^{-1}$.

${ }^{1} \mathrm{H} \mathrm{NMR}\left(\mathrm{CDCl}_{3}, 400 \mathrm{MHz}\right): \delta=7.28-7.17(\mathrm{~m}, 5 \mathrm{H}), 4.98(\mathrm{~d}, J=8.0 \mathrm{~Hz}$, $1 \mathrm{H}), 4.65(\mathrm{~d}, J=8.0 \mathrm{~Hz}, 1 \mathrm{H}), 3.91-3.89(\mathrm{~m}, 1 \mathrm{H}), 3.63-3.38(\mathrm{~m}, 4 \mathrm{H})$, 3.18 (br s, $1 \mathrm{H}), 2.88$ (d, J = 8.0 Hz, $2 \mathrm{H}), 1.37(\mathrm{~s}, 9 \mathrm{H})$.

${ }^{13} \mathrm{C}$ NMR $\left(\mathrm{CDCl}_{3}, 125 \mathrm{MHz}\right): \delta=156.9,138.0,129.5,129.3,128.6$, 126.5, 80.5, 80.1, 73.2, 71.6, 64.0, 59.6, 52.6, 38.2, 31.3, 29.8, 28.4.

HRMS (ESI-TOF): $m / z[\mathrm{M}+\mathrm{Na}]^{+}$calcd for $\mathrm{C}_{15} \mathrm{H}_{23} \mathrm{NNaO}_{4}: 304.1525$; found: 304.1523 .

\section{tert-Butyl ((2R,3R)-3,4-Dihydroxy-1-phenylbutan-2-yl)carbamate} (5b)

Column chromatography (petroleum ether/EtOAc, 60:40).

Yield: $0.69 \mathrm{~g}$ (64\%); clear oil; $[\alpha]_{\mathrm{D}}{ }^{27}-8.59$ (c $0.74, \mathrm{CHCl}_{3}$ ).

IR (thin film): 3360, 2978, 2928, 1686, $1524 \mathrm{~cm}^{-1}$.

${ }^{1} \mathrm{H} \mathrm{NMR}\left(\mathrm{CDCl}_{3}, 500 \mathrm{MHz}\right): \delta=7.31-7.22(\mathrm{~m}, 5 \mathrm{H}), 4.82(\mathrm{~d}, J=5.0 \mathrm{~Hz}$, $1 \mathrm{H}), 4.56(\mathrm{~d}, J=5.0 \mathrm{~Hz}, 1 \mathrm{H}), 3.86-3.81(\mathrm{~m}, 1 \mathrm{H}), 3.68-3.36(\mathrm{~m}, 4 \mathrm{H})$, 3.11-3.08 (m, $1 \mathrm{H}), 2.92-2.88(\mathrm{~m}, 2 \mathrm{H}), 1.38(\mathrm{~s}, 9 \mathrm{H})$.

${ }^{13} \mathrm{C}$ NMR $\left(\mathrm{CDCl}_{3}, 125 \mathrm{MHz}\right): \delta=157.2,137.4,129.5,128.8,126.8,80.6$, 73.2, 63.0, 52.4, 36.6, 31.7, 29.8, 28.3.

HRMS (ESI-TOF): $m / z[\mathrm{M}+\mathrm{Na}]^{+}$calcd for $\mathrm{C}_{15} \mathrm{H}_{23} \mathrm{NNaO}_{4}: 304.1525$; found: 304.1528 .

tert-Butyl (4R,5S)-4-Benzyl-5-((R)-2,3-dihydroxypropyl)-2,2-dimethyloxazolidine-3-carboxylate (19)

Column chromatography (petroleum ether/EtOAc, 50:50).

Yield: $0.68 \mathrm{~g}$ (65\%); clear oil; $[\alpha]_{\mathrm{D}}{ }^{27}+11.94$ (c $0.92, \mathrm{CHCl}_{3}$ ).

IR (thin film): 3418, 3063, 3029, 2924, 2855, 1694, 1682, $1604 \mathrm{~cm}^{-1}$.

${ }^{1} \mathrm{H}$ NMR $\left(\mathrm{CDCl}_{3}, 500 \mathrm{MHz}\right): \delta=7.27-7.18(\mathrm{~m}, 5 \mathrm{H}), 4.29-4.10(\mathrm{~m}, 2 \mathrm{H})$, 3.66 (br s, 1 H), 3.46-3.23 (m, 2 H), 2.97-2.82 (m, 2 H), 1.82-1.67 (m, $3 \mathrm{H}), 1.57-1.53(\mathrm{~m}, 6 \mathrm{H}), 1.44,1.34(\mathrm{~s}, 9 \mathrm{H})$.

${ }^{13} \mathrm{C}$ NMR $\left(\mathrm{CDCl}_{3}, 125 \mathrm{MHz}\right): \delta=151.9,151.6,138.7,129.4,129.3$, 128.6, 128.4, 126.4, 126.2, 93.6, 92.9, 80.4, 80.0, 76.1, 71.2, 71.1, 66.3, $61.1,60.9,36.7,36.0,32.9,29.8,28.4,28.1,27.5,26.8,25.2,24.0$.

HRMS (ESI-TOF): $m / z[\mathrm{M}+\mathrm{Na}]^{+}$calcd for $\mathrm{C}_{20} \mathrm{H}_{31} \mathrm{NNaO}_{5}: 388.2100$; found: 388.2100 .

\section{Silyl Protection; General Procedure}

Compound 5 ( $1.00 \mathrm{~g}, 3.55 \mathrm{mmol}$ ) was dissolved in anhydrous DCM $(20 \mathrm{~mL})$ and the solution cooled to $0{ }^{\circ} \mathrm{C}$. TBDPSCl $(1.07 \mathrm{~mL}, 3.91$ $\mathrm{mmol})$, DMAP $(0.08 \mathrm{~g}, 0.71 \mathrm{mmol})$ and triethylamine $(0.74 \mathrm{~mL}, 5.32$ $\mathrm{mmol}$ ) were added and the reaction mixture was stirred at r.t. for $8 \mathrm{~h}$. On complete disappearance of starting material, the reaction was quenched with saturated aqueous citric acid $(20 \mathrm{~mL})$, the crude product was extracted with DCM $(2 \times 30 \mathrm{~mL})$ and the combined organic phases containing crude product were dried over $\mathrm{Na}_{2} \mathrm{SO}_{4}$, filtered, concentrated under vacuum, and purified by column chromatography.

tert-butyl ((2R,3S)-4-((tert-Butyldiphenylsilyl)oxy)-3-hydroxy-1phenylbutan-2-yl)carbamate (6a)

Column chromatography (petroleum ether/EtOAc, 80:20).
Yield: $1.51 \mathrm{~g}(82 \%)$; clear oil; $[\alpha]_{\mathrm{D}}{ }^{27}+17.28$ (c $\left.0.96, \mathrm{CHCl}_{3}\right)$. IR (thin film): 3434, 3070, 3027, 2927, 2856, $1689 \mathrm{~cm}^{-1}$.

${ }^{1} \mathrm{H}$ NMR $\left(\mathrm{CDCl}_{3}, 400 \mathrm{MHz}\right): \delta=7.62-7.57(\mathrm{~m}, 4 \mathrm{H}), 7.42-7.21(\mathrm{~m}$, $11 \mathrm{H}), 4.93$ (br s, $1 \mathrm{H}), 3.76-3.69$ (m, $2 \mathrm{H}), 3.61-3.60$ (m, $2 \mathrm{H}), 2.96-$ 2.85 (m, 2 H), 2.67 (br s, 1 H), 1.35 (s, 9 H), 1.04 (s, 9 H).

${ }^{13} \mathrm{C}$ NMR $\left(\mathrm{CDCl}_{3}, 100 \mathrm{MHz}\right): \delta=155.9,138.4,135.6,133.1,130.0$, $129.5,128.5,127.9,126.4,79.4,71.1,65.7,52.7,38.6,29.8,28.4,27.0$, 19.3.

HRMS (ESI-TOF): $m / z[\mathrm{M}+\mathrm{Na}]^{+}$calcd for $\mathrm{C}_{31} \mathrm{H}_{41} \mathrm{NNaO}_{4} \mathrm{Si}: 542.2703$; found: 542.2700

tert-Butyl ((2R,3R)-4-((tert-Butyldiphenylsilyl)oxy)-3-hydroxy-1phenylbutan-2-yl)carbamate (6b)

Column chromatography (petroleum ether/EtOAc, 80:20).

Yield: $1.55 \mathrm{~g}$ (84\%); clear oil; $[\alpha]_{\mathrm{D}}{ }^{27}+2.26$ (c $1.45, \mathrm{CHCl}_{3}$ ).

IR (thin film): 3417, 2930, 2857, 1692, $1497 \mathrm{~cm}^{-1}$.

${ }^{1} \mathrm{H} \mathrm{NMR}\left(\mathrm{CDCl}_{3}, 500 \mathrm{MHz}\right): \delta=7.70-7.68(\mathrm{~m}, 3 \mathrm{H}), 7.46-7.39(\mathrm{~m}, 6 \mathrm{H})$, 7.29-7.17 (m, 6 H), 4.98 (br s, 1 H), 3.99 (br s, 1 H), 3.76-3.62 (m, $3 \mathrm{H}$ ), 3.08 (br s, $1 \mathrm{H}), 2.96-2.85(\mathrm{~m}, 2 \mathrm{H}), 1.36(\mathrm{~s}, 9 \mathrm{H}), 1.11(\mathrm{~s}, 9 \mathrm{H})$.

${ }^{13} \mathrm{C}$ NMR $\left(\mathrm{CDCl}_{3}, 125 \mathrm{MHz}\right): \delta=156.0,138.0,135.7,132.9,132.8$, 130.0, 129.5, 128.5, 128.0, 127.9, 126.4, 79.4, 72.6, 65.4, 54.3, 36.6, 29.8, 28.4, 27.0, 19.3 .

HRMS (ESI-TOF): $m / z[\mathrm{M}+\mathrm{Na}]^{+}$calcd for $\mathrm{C}_{31} \mathrm{H}_{41} \mathrm{NNaO}_{4} \mathrm{Si}: 542.2703$; found: 542.2705 .

\section{MOM Protection; General Procedure}

MOM chloride ( $0.58 \mathrm{~mL}, 7.68 \mathrm{mmol}$ ) followed by Hunig's base, DIPEA $(1.68 \mathrm{~mL}, 9.62 \mathrm{mmol}$ ) were added to a stirred solution of compound 6 $(1.00 \mathrm{~g}, 1.92 \mathrm{mmol})$ in $\mathrm{DCM}(25 \mathrm{~mL})$ at $0{ }^{\circ} \mathrm{C}$, and the mixture was stirred vigorously at r.t. for $6 \mathrm{~h}$. On complete disappearance of starting material, the reaction was quenched with water $(20 \mathrm{~mL})$, and the mixture was extracted with DCM $(2 \times 30 \mathrm{~mL})$ and the combined organic phases were washed with $2 \% \mathrm{HCl}(2 \times 20 \mathrm{~mL})$, dried over $\mathrm{Na}_{2} \mathrm{SO}_{4}$, filtered, concentrated and purified through column chromatography.

tert-Butyl ((2R,3S)-4-((tert-Butyldiphenylsilyl)oxy)-3-(methoxymethoxy)-1-phenylbutan-2-yl)carbamate (7a)

Column chromatography (petroleum ether/EtOAc, 85:15).

Yield: $0.84 \mathrm{~g}$ (78\%); clear oil; $[\alpha]_{D}{ }^{27}+1.65$ (c $0.48, \mathrm{CHCl}_{3}$ ).

IR (thin film): 2928, 2856, 1715, $1494 \mathrm{~cm}^{-1}$.

${ }^{1} \mathrm{H}$ NMR $\left(\mathrm{CDCl}_{3}, 400 \mathrm{MHz}\right): \delta=7.53-7.47(\mathrm{~m}, 4 \mathrm{H}), 7.35-7.15(\mathrm{~m}$, $11 \mathrm{H}), 4.93(\mathrm{~d}, J=8.0 \mathrm{~Hz}, 1 \mathrm{H}), 4.58-4.43(\mathrm{~m}, 2 \mathrm{H}), 4.09-4.04(\mathrm{~m}, 1 \mathrm{H})$, 3.56-3.51 (m, $3 \mathrm{H}), 3.28$ (s, $3 \mathrm{H}), 2.88-2.71(\mathrm{~m}, 2 \mathrm{H}), 1.33(\mathrm{~s}, 9 \mathrm{H}), 0.91$ (s, $9 \mathrm{H})$.

${ }^{13} \mathrm{C}$ NMR $\left(\mathrm{CDCl}_{3}, 100 \mathrm{MHz}\right): \delta=155.5,135.6,133.2,129.7,129.6$, 128.5, 127.8, 126.3, 97.1, 79.1, 63.6, 55.9, 52.4, 38.7, 28.5, 26.8, 19.2 .

HRMS (ESI-TOF): $m / z[\mathrm{M}+\mathrm{H}]^{+}$calcd for $\mathrm{C}_{33} \mathrm{H}_{46} \mathrm{NO}_{5} \mathrm{Si}: 564.3145$; found: 564.3141

tert-Butyl ((2R,3R)-4-((tert-Butyldiphenylsilyl)oxy)-3-(methoxymethoxy)-1-phenylbutan-2-yl)carbamate (7b)

Column chromatography (petroleum ether/EtOAc, 85:15).

Yield: $0.86 \mathrm{~g}$ (80\%); clear oil; $[\alpha]_{\mathrm{D}}{ }^{27}+11.05$ (c 2.63, $\mathrm{CHCl}_{3}$ ).

IR (thin film): 3070, 3027, 2930, 2891, 2857, 1713, 1603, $1589 \mathrm{~cm}^{-1}$. 
${ }^{1} \mathrm{H} \mathrm{NMR}\left(\mathrm{CDCl}_{3}, 400 \mathrm{MHz}\right): \delta=7.70-7.68(\mathrm{~m}, 4 \mathrm{H}), 7.45-7.38(\mathrm{~m}, 6 \mathrm{H})$, 7.25-7.16 (m, $5 \mathrm{H}), 5.38$ (d, J = $12.0 \mathrm{~Hz}, 1 \mathrm{H}), 4.65$ (br s, $2 \mathrm{H}), 4.17$ (d, $J=8.0 \mathrm{~Hz}, 1 \mathrm{H}), 3.84-3.80(\mathrm{~m}, 1 \mathrm{H}), 3.71-3.61(\mathrm{~m}, 2 \mathrm{H}), 3.32(\mathrm{~s}, 3 \mathrm{H})$, $2.82(\mathrm{~d}, J=8.0 \mathrm{~Hz}, 2 \mathrm{H}), 1.35(\mathrm{~s}, 9 \mathrm{H}), 1.08(\mathrm{~s}, 9 \mathrm{H})$.

${ }^{13} \mathrm{C}$ NMR $\left(\mathrm{CDCl}_{3}, 125 \mathrm{MHz}\right): \delta=155.6,138.4,135.7,135.7,133.0$, 129.9, 129.9, 129.2, 128.3, 127.8, 127.8, 126.2, 96.6, 93.6, 78.7, 64.4, 63.5, 55.7, 52.9, 38.6, 36.9, 28.4, 26.9, 19.2 .

HRMS (ESI-TOF): $m / z[\mathrm{M}+\mathrm{H}]^{+}$calcd for $\mathrm{C}_{33} \mathrm{H}_{46} \mathrm{NO}_{5} \mathrm{Si}: 564.3145$; found: 564.3149 .

\section{Silyl Deprotection; General Procedure}

TBAF (1 M in THF, $1.94 \mathrm{~mL}, 1.94 \mathrm{mmol}$ ) was added to a stirred solution of compound $7(1.00 \mathrm{~g}, 1.77 \mathrm{mmol})$ in anhydrous THF $(15 \mathrm{~mL})$ at $0{ }^{\circ} \mathrm{C}$ and the solution was stirred at r.t. for $2 \mathrm{~h}$. On complete disappearance of starting material, the reaction was quenched with saturated aqueous $\mathrm{NH}_{4} \mathrm{Cl}(30 \mathrm{~mL})$ and the mixture was extracted with EtOAc $(2 \times 30 \mathrm{~mL})$. The combined organic phases were dried over $\mathrm{Na}_{2}-$ $\mathrm{SO}_{4}$, filtered, concentrated under vacuum, and purified by column chromatography.

tert-Butyl ((2R,3S)-4-Hydroxy-3-(methoxymethoxy)-1-phenylbutan-2-yl)carbamate (8a)

Column chromatography (petroleum ether/EtOAc, 70:30).

Yield: $0.51 \mathrm{~g}$ (89\%); clear oil; $[\alpha]_{\mathrm{D}}{ }^{27}+42.55$ (c $0.79, \mathrm{CHCl}_{3}$ ).

IR (thin film): 3444, 3063, 3028, 2927, 2854, 1693, $1604 \mathrm{~cm}^{-1}$.

$\left.{ }^{1} \mathrm{H} \mathrm{NMR} \mathrm{(CDCl}, 500 \mathrm{MHz}\right): \delta=7.23-7.12(\mathrm{~m}, 5 \mathrm{H}), 4.71(\mathrm{~d}, J=10.0 \mathrm{~Hz}$, $1 \mathrm{H}), 4.67-4.53(\mathrm{~m}, 2 \mathrm{H}), 4.05$ (dd, $J=15.0,10.0 \mathrm{~Hz}, 1 \mathrm{H}), 3.61$ (dd, $J=$ 10.0, $5.0 \mathrm{~Hz}, 1 \mathrm{H}), 3.46-3.44(\mathrm{~m}, 1 \mathrm{H}), 3.41-3.37(\mathrm{~m}, 1 \mathrm{H}), 3.34(\mathrm{~s}, 3 \mathrm{H})$, 2.84-2.74 (m, $2 \mathrm{H}), 1.33(\mathrm{~s}, 9 \mathrm{H})$.

${ }^{13} \mathrm{C} \mathrm{NMR}\left(\mathrm{CDCl}_{3}, 125 \mathrm{MHz}\right): \delta=156.6,137.9,129.1,128.6,126.6,97.7$, $80.7,80.0,62.6,55.9,52.1,38.3,29.8,28.4$.

HRMS (ESI- TOF): $m / z[\mathrm{M}+\mathrm{Na}]^{+}$calcd for $\mathrm{C}_{17} \mathrm{H}_{27} \mathrm{NNaO}_{5}: 348.1787$; found: 348.1788 .

\section{tert-Butyl ((2R,3R)-4-Hydroxy-3-(methoxymethoxy)-1-phenyl-} butan-2-yl)carbamate (8b)

Column chromatography (petroleum ether/EtOAc, 70:30).

Yield: $0.52 \mathrm{~g}$ (90\%); clear oil; $[\alpha]_{\mathrm{D}}{ }^{27}+0.47$ (c 1.02, $\mathrm{CHCl}_{3}$ ).

IR (thin film): 3471, 3368, 3021, 2964, 2929, 1692, $1523 \mathrm{~cm}^{-1}$.

${ }^{1} \mathrm{H} \mathrm{NMR}\left(\mathrm{CDCl}_{3}, 500 \mathrm{MHz}\right): \delta=7.28-7.18(\mathrm{~m}, 5 \mathrm{H}), 4.80-4.70(\mathrm{~m}, 3 \mathrm{H})$, 4.04 (br s, $1 \mathrm{H}$ ), 3.70-3.66 (m, $2 \mathrm{H}$ ), 3.50 (br s, $1 \mathrm{H}), 3.45$ (s, $3 \mathrm{H}), 3.03-$ 3.00 (m, $1 \mathrm{H}), 2.75-2.70$ (m, $1 \mathrm{H}), 1.89$ (br s, $1 \mathrm{H}), 1.32$ (s, $9 \mathrm{H})$.

${ }^{13} \mathrm{C} \mathrm{NMR}\left(\mathrm{CDCl}_{3}, 125 \mathrm{MHz}\right): \delta=156.0,137.9,129.3,128.5,126.5,97.0$, $82.4,79.7,62.3,56.0,52.0,36.7,29.7,28.3$.

HRMS (ESI-TOF): $m / z[\mathrm{M}+\mathrm{H}]^{+}$calcd for $\mathrm{C}_{17} \mathrm{H}_{28} \mathrm{NO}_{5}: 326.1967$; found: 326.1968

\section{Oxidation of Primary Alcohols; General Procedure}

Pyridinium dichromate $(11.56 \mathrm{~g}, 30.75 \mathrm{mmol})$ was added to the stirred solution of alcohol $8(1.00 \mathrm{~g}, 3.07 \mathrm{mmol})$ in DMF $(30 \mathrm{~mL})$ and stirring was continued at r.t. for $8 \mathrm{~h}$. On complete disappearance of the starting material, the reaction was quenched with water $(300 \mathrm{~mL})$ and extracted with $\mathrm{Et}_{2} \mathrm{O}(2 \times 50 \mathrm{~mL})$. The combined organic phases were washed with saturated aqueous $\mathrm{NaHCO}_{3}(2 \times 30 \mathrm{~mL})$ and the aqueous extracts containing the carboxylate salts were combined and acidified with saturated aqueous $\mathrm{KHSO}_{4}(2 \times 50 \mathrm{~mL})$ and this was ex- tracted with $\mathrm{Et}_{2} \mathrm{O}(2 \times 50 \mathrm{~mL})$. The ether layers were combined, dried over $\mathrm{Na}_{2} \mathrm{SO}_{4}$, filtered, concentrated, and purified by column chromatography.

(2S,3R)-3-((tert-Butoxycarbonyl)amino)-2-(methoxymethoxy)-4phenylbutanoic Acid (9a)

Column chromatography (DCM/MeOH, 95:5).

Yield: $0.79 \mathrm{~g}$ (76\%); clear oil; $[\alpha]_{\mathrm{D}}^{27}+9.18$ (c $0.29, \mathrm{CHCl}_{3}$ ).

IR (thin film): 3334, 2924, 2853, 1715, $1497 \mathrm{~cm}^{-1}$.

${ }^{1} \mathrm{H}$ NMR $\left(\mathrm{CDCl}_{3}, 500 \mathrm{MHz}\right): \delta=8.03(\mathrm{~s}, 1 \mathrm{H}), 7.30-7.19(\mathrm{~m}, 5 \mathrm{H}), 5.09$ $(\mathrm{d}, J=8.0 \mathrm{~Hz}, 1 \mathrm{H}), 4.77-4.70(\mathrm{~m}, 2 \mathrm{H}), 4.37(\mathrm{~d}, J=4.0 \mathrm{~Hz}, 1 \mathrm{H}), 4.17(\mathrm{~s}$, $1 \mathrm{H}), 3.46$ (s, $3 \mathrm{H}), 2.90-2.88(\mathrm{~m}, 2 \mathrm{H}), 1.34(\mathrm{~s}, 9 \mathrm{H})$.

${ }^{13} \mathrm{C}$ NMR $\left(\mathrm{CDCl}_{3}, 125 \mathrm{MHz}\right): \delta=173.1,163.2,155.6,137.5,129.4$, 128.7, 126.7, 96.8, 80.1, 75.1, 56.7, 54.0, 38.5, 29.8, 28.3.

HRMS (ESI-TOF): $m / z[\mathrm{M}+\mathrm{Na}]^{+}$calcd for $\mathrm{C}_{17} \mathrm{H}_{25} \mathrm{NNaO}_{6}: 362.1580$; found: 362.1558 .

(2R,3R)-3-((tert-Butoxycarbonyl)amino)-2-(methoxymethoxy)-4phenylbutanoic Acid (9b)

Column chromatography (DCM/MeOH, 95:5).

Yield: $0.80 \mathrm{~g}\left(78 \%\right.$ ); clear oil; $[\alpha]_{\mathrm{D}}{ }^{27}+48.55$ (c $0.41, \mathrm{CHCl}_{3}$ ).

IR (thin film): 3395, 2924, 2853, 1692, 1603, $1497 \mathrm{~cm}^{-1}$.

${ }^{1} \mathrm{H}$ NMR $\left(\mathrm{CDCl}_{3}, 400 \mathrm{MHz}\right): \delta=7.30-7.19(\mathrm{~m}, 5 \mathrm{H}), 5.07(\mathrm{~d}, J=8.0 \mathrm{~Hz}$, $1 \mathrm{H}), 4.76-4.69(\mathrm{~m}, 2 \mathrm{H}), 4.37$ (d, J = 8.0 Hz, $1 \mathrm{H}), 4.16$ (br s, $1 \mathrm{H}), 3.45$ (s, $3 \mathrm{H}), 2.88(\mathrm{~d}, J=8.0 \mathrm{~Hz}, 2 \mathrm{H}), 1.33(\mathrm{~s}, 9 \mathrm{H})$.

${ }^{13} \mathrm{C}$ NMR $\left(\mathrm{CDCl}_{3}, 100 \mathrm{MHz}\right): \delta=173.4,163.4,155.3,137.5,129.3$, 128.3, 126.4, 96.6, 79.6, 56.2, 53.3, 36.9, 36.0, 31.8, 29.7, 28.2, 28.0 .

HRMS (ESI-TOF): $m / z[\mathrm{M}+\mathrm{Na}]^{+}$calcd for $\mathrm{C}_{17} \mathrm{H}_{25} \mathrm{NNaO}_{6}: 362.1580$; found: 362.1584

\section{Peptide Coupling of 9}

Compound 9 ( $0.19 \mathrm{~g}, 0.55 \mathrm{mmol}$ ) was dissolved in anhydrous DCM $(10 \mathrm{~mL})$ and the solution was cooled in an ice bath followed by addition of $\mathrm{EDC} \cdot \mathrm{HCl}(0.21 \mathrm{~g}, 1.12 \mathrm{mmol})$ and $\mathrm{HOBt}(0.15 \mathrm{~g}, 1.12 \mathrm{mmol})$ and then stirred for $20 \mathrm{~min}$. H-Leu-OBn $(0.17 \mathrm{~g}, 0.55 \mathrm{mmol})$ was added to the reaction mixture followed by DIPEA $(0.20 \mathrm{~mL}, 1.23 \mathrm{mmol})$ and the mixture was stirred at r.t. for $6 \mathrm{~h}$. On complete disappearance of starting material, the organic layer was washed with aqueous citric acid ( 3 $\times 15 \mathrm{~mL}$ ) and $2 \mathrm{M}$ aqueous $\mathrm{NaHCO}_{3}(3 \times 15 \mathrm{~mL})$. The organic layers were combined, dried over $\mathrm{Na}_{2} \mathrm{SO}_{4}$, filtered, concentrated, and purified by column chromatography.

Benzyl ((2S,3R)-3-((tert-Butoxycarbonyl)amino)-2-(methoxymethoxy)-4-phenylbutanoyl)-L-leucinate (10a)

Column chromatography (petroleum ether/EtOAc, 70:30).

Yield: $0.24 \mathrm{~g}$ (82\%); white solid; $[\alpha]_{\mathrm{D}}^{27}+32.23$ (c 0.69, $\mathrm{CHCl}_{3}$ ); mp 99$101{ }^{\circ} \mathrm{C}$.

IR (thin film): 3333, 3277, 3063, 3030, 2961, 2929, 2873, 1748, 1688, $1650,1547,1524 \mathrm{~cm}^{-1}$

${ }^{1} \mathrm{H} \mathrm{NMR}\left(\mathrm{CDCl}_{3}, 400 \mathrm{MHz}\right): \delta=7.31-7.09(\mathrm{~m}, 10 \mathrm{H}), 6.96(\mathrm{~d}, J=8.7 \mathrm{~Hz}$, $1 \mathrm{H}), 5.19$ (d, $J=9.9 \mathrm{~Hz}, 1 \mathrm{H}), 5.11-5.04(\mathrm{~m}, 2 \mathrm{H}), 4.69-4.62(\mathrm{~m}, 3 \mathrm{H})$, 4.17 (br s, $1 \mathrm{H}), 4.06(\mathrm{~m}, 1 \mathrm{H}), 3.35(\mathrm{~s}, 3 \mathrm{H}), 2.82$ (dd, $J=13.7,5.4 \mathrm{~Hz}$, $1 \mathrm{H}), 2.59-2.54(\mathrm{~m}, 1 \mathrm{H}), 1.62-1.50(\mathrm{~m}, 3 \mathrm{H}), 1.23(\mathrm{~s}, 9 \mathrm{H}), 0.86(\mathrm{~d}, J=$ $4.3 \mathrm{~Hz}, 6 \mathrm{H})$.

${ }^{13} \mathrm{C}$ NMR $\left(\mathrm{CDCl}_{3}, 125 \mathrm{MHz}\right): \delta=172.5,170.5,155.0,137.8,135.3$, 129.4, 128.7, 128.6, 128.4, 128.4, 126.5, 115.5, 96.9, 79.2, 78.1, 67.2, $56.7,53.3,50.4,41.5,37.5,29.8,28.3,24.9,22.9,21.8$. 
HRMS (ESI-TOF): $m / z[\mathrm{M}+\mathrm{H}]^{+}$calcd for $\mathrm{C}_{30} \mathrm{H}_{43} \mathrm{~N}_{2} \mathrm{O}_{7}$ : 543.3070; found: 543.3079.

Benzyl ((2R,3R)-3-((tert-Butoxycarbonyl)amino)-2-(methoxymethoxy)-4-phenylbutanoyl)-L-leucinate (10b)

Column chromatography (petroleum ether/EtOAc, 70:30).

Yield: $0.24 \mathrm{~g}$ (82\%); white solid; $[\alpha]_{\mathrm{D}}{ }^{27}+14.68\left(\mathrm{c} 0.68, \mathrm{CHCl}_{3}\right)$; mp 98$99{ }^{\circ} \mathrm{C}$.

IR (thin film): 3348, 3306, 3030, 2957, 2929, 1738, 1693, 1654, 1524 $\mathrm{cm}^{-1}$.

${ }^{1} \mathrm{H} \mathrm{NMR}\left(\mathrm{CDCl}_{3}, 500 \mathrm{MHz}\right): \delta=7.33-7.17(\mathrm{~m}, 10 \mathrm{H}), 6.87(\mathrm{~d}, J=5.0 \mathrm{~Hz}$, $1 \mathrm{H}), 5.22-5.13(\mathrm{~m}, 3 \mathrm{H}), 4.71-4.61(\mathrm{~m}, 3 \mathrm{H}), 4.34-4.23(\mathrm{~m}, 2 \mathrm{H}), 3.37$ (s, $3 \mathrm{H}), 2.93-2.55(\mathrm{~m}, 2 \mathrm{H}), 1.73-1.56(\mathrm{~m}, 3 \mathrm{H}), 1.34(\mathrm{~s}, 9 \mathrm{H}), 0.93(\mathrm{~d}$, $J=5.0 \mathrm{~Hz}, 6 \mathrm{H})$.

${ }^{13} \mathrm{C}$ NMR $\left(\mathrm{CDCl}_{3}, 125 \mathrm{MHz}\right): \delta=172.6,169.9,155.6,138.0,135.4$, 129.5, 128.7, 128.5, 128.4, 126.4, 96.9, 79.3, 78.8, 67.3, 56.4, 54.1, $50.7,40.8,36.8,29.8,28.4,25.1,22.9,21.8$.

HRMS (ESI-TOF): $m / z$ [M $+\mathrm{H}]^{+}$calcd for $\mathrm{C}_{30} \mathrm{H}_{43} \mathrm{~N}_{2} \mathrm{O}_{7}$ : 543.3070; found: 543.3079 .

\section{Procedure for Hydrogenolysis of $\mathbf{1 0}$}

To a stirred solution of $\mathbf{1 0}(0.13 \mathrm{~g}, 0.24 \mathrm{mmol})$ in anhydrous $\mathrm{MeOH}$ (10 $\mathrm{mL}), \mathrm{Pd} / \mathrm{C}(10 \mathrm{~mol} \%)$ was added and the mixture was stirred vigorously for $3 \mathrm{~h}$ at r.t. under $\mathrm{H}_{2}$. On complete disappearance of starting material, the reaction mixture was filtered through a Celite ${ }^{\circledR}$ pad, solvent was removed under vacuum and the residue was purified by column chromatography.

((2S,3R)-3-((tert-Butoxycarbonyl)amino)-2-(methoxymethoxy)-4phenylbutanoyl)-L-leucine (11a)

Column chromatography $\left(\mathrm{CH}_{2} \mathrm{Cl}_{2} / \mathrm{MeOH}, 95: 5\right)$.

Yield: $0.10 \mathrm{~g}$ (92\%); clear oil; $[\alpha]_{\mathrm{D}}^{27}+21.11$ (c $0.36, \mathrm{CHCl}_{3}$ ).

IR (thin film): 3333, 2925, 2854, 1714, 1529, $1454 \mathrm{~cm}^{-1}$.

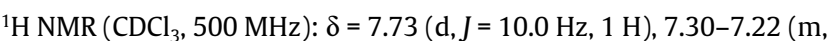
$5 \mathrm{H}), 6.84(\mathrm{~d}, J=5.0 \mathrm{~Hz}, 1 \mathrm{H}), 4.88-4.75(\mathrm{~m}, 2 \mathrm{H}), 4.56(\mathrm{~d}, J=5.2 \mathrm{~Hz}$, $1 \mathrm{H}), 4.14-4.08$ (m, $2 \mathrm{H}), 3.47$ (s, $3 \mathrm{H}), 2.98-2.86(\mathrm{~m}, 2 \mathrm{H}), 1.73-1.54$ $(\mathrm{m}, 3 \mathrm{H}), 1.29(\mathrm{~s}, 9 \mathrm{H}), 0.92-0.89(\mathrm{~m}, 6 \mathrm{H})$.

${ }^{13} \mathrm{C}$ NMR $\left(\mathrm{CDCl}_{3}, 125 \mathrm{MHz}\right): \delta=176.4,169.6,157.5,137.8,129.6$, 128.8, 126.8, 97.1, 81.8, 78.0, 57.1, 56.1, 50.5, 43.3, 39.7, 29.8, 28.1, $25.0,22.8,22.4$.

HRMS (ESI-TOF): $m / z$ [M $+\mathrm{Na}]^{+}$calcd for $\mathrm{C}_{23} \mathrm{H}_{36} \mathrm{~N}_{2} \mathrm{NaO}_{7}: 475.2420$; found: 475.2452 .

((2R,3R)-3-((tert-Butoxycarbonyl)amino)-2-(methoxymethoxy)-4phenylbutanoyl)-L-leucine (11b)

Column chromatography (DCM/MeOH, 95:5).

Yield: $0.10 \mathrm{~g}$ (92\%); clear oil; $[\alpha]_{\mathrm{D}}{ }^{27}+25.37$ (c $0.66, \mathrm{CHCl}_{3}$ ).

IR (thin film): 3300, 2954, 2740, 1730, $1520 \mathrm{~cm}^{-1}$.

${ }^{1} \mathrm{H} \mathrm{NMR}\left(\mathrm{CDCl}_{3}, 400 \mathrm{MHz}\right): \delta=7.25-7.10(\mathrm{~m}, 5 \mathrm{H}), 5.24$ (br s, $\left.1 \mathrm{H}\right)$, $4.63(\mathrm{~m}, 2 \mathrm{H}), 4.31-4.14(\mathrm{~m}, 2 \mathrm{H}), 3.35(\mathrm{~s}, 3 \mathrm{H}), 2.94-2.73(\mathrm{~m}, 2 \mathrm{H})$, 1.79-1.53 (m, $3 \mathrm{H}), 1.24(\mathrm{~s}, 9 \mathrm{H}), 0.93-0.83(\mathrm{~m}, 6 \mathrm{H})$.

${ }^{13} \mathrm{C}$ NMR $\left(\mathrm{CDCl}_{3}, 100 \mathrm{MHz}\right): \delta=175.7,175.4,170.6,169.6,157.3$, 155.8, 138.3, 137.9, 129.6, 129.4, 128.4, 126.4, 96.6, 96.4, 81.1, 79.5, 79.0, 78.4, 56.4, 55.8, 54.2, 50.7, 50.4, 41.2, 37.4, 36.7, 32.0, 29.8, 28.3, $28.0,25.1,23.1,22.8,21.7,14.2$.

HRMS (ESI-TOF): $m / z$ [M $+\mathrm{Na}]^{+}$calcd for $\mathrm{C}_{23} \mathrm{H}_{36} \mathrm{~N}_{2} \mathrm{NaO}_{7}: 475.2420$; found: 475.2452 .

\section{Acidolysis Reaction; General Procedure}

$\mathrm{HCl}(6 \mathrm{M}$ in EtOAc, $0.50 \mathrm{~mL})$ was added to $11(0.083 \mathrm{~g}, 0.18 \mathrm{mmol}), 14$ $(0.092 \mathrm{~g}, 0.15 \mathrm{mmol})$ or $21(0.050 \mathrm{~g}, 0.14 \mathrm{mmol})$ at $0{ }^{\circ} \mathrm{C}$ and the mixture was stirred at r.t. for $4 \mathrm{~h}$. On complete disappearance of starting material, solvent was removed under vacuum and the white residual solid was triturated 3 to 4 times with cold EtOAc ( $5 \mathrm{~mL}$ ).

((2S,3R)-3-Amino-2-hydroxy-4-phenylbutanoyl)-L-leucine (1a) Yield: $0.058 \mathrm{~g}$ (94\%); white solid; $[\alpha]_{\mathrm{D}}{ }^{27}-15.83$ (c $\left.0.24, \mathrm{CH}_{3} \mathrm{OH}\right) ; \mathrm{mp}$ $212-215{ }^{\circ} \mathrm{C}\left\{\right.$ lit. $^{19}[\alpha]_{\mathrm{D}}{ }^{20}-15.2$ (c $\left.\left.0.83,1 \mathrm{M} \mathrm{HCl}\right) ; \operatorname{mp} 210-214{ }^{\circ} \mathrm{C}\right\}$.

IR (thin film): 3737, 2953, 1725, 1660, 1555, 1518, $1492 \mathrm{~cm}^{-1}$.

${ }^{1} \mathrm{H}$ NMR $\left(\mathrm{D}_{2} \mathrm{O}, 500 \mathrm{MHz}\right): \delta=7.48-7.36(\mathrm{~m}, 5 \mathrm{H}), 4.41(\mathrm{dd}, J=10.0$, $5.1 \mathrm{~Hz}, 1 \mathrm{H}), 4.33(\mathrm{~d}, J=5.0 \mathrm{~Hz}, 1 \mathrm{H}), 3.89-3.85(\mathrm{~m}, 1 \mathrm{H}), 3.19(\mathrm{dd}, J=$ 15.0, $5.0 \mathrm{~Hz}, 1 \mathrm{H}), 2.97(\mathrm{dd}, J=15.0,10.0 \mathrm{~Hz}, 1 \mathrm{H}), 1.79-1.67(\mathrm{~m}, 3 \mathrm{H})$, $0.97(\mathrm{~d}, J=5.0 \mathrm{~Hz}, 3 \mathrm{H}), 0.94(\mathrm{~d}, J=5.0 \mathrm{~Hz}, 3 \mathrm{H})$.

${ }^{13} \mathrm{C}$ NMR $\left(\mathrm{D}_{2} \mathrm{O}, 125 \mathrm{MHz}\right): \delta=176.3,172.7,134.9,129.4,129.2,127.7$, 69.5, 54.9, 51.6, 39.1, 34.8, 24.5, 22.0, 20.7.

HRMS (ESI-TOF): $m / z[\mathrm{M}+\mathrm{H}]^{+}$calcd for $\mathrm{C}_{16} \mathrm{H}_{25} \mathrm{~N}_{2} \mathrm{O}_{4}$ : 309.1814; found: 309.1811.

((2R,3R)-3-Amino-2-hydroxy-4-phenylbutanoyl)-L-leucine (1b)

Yield: $0.060 \mathrm{~g}(97 \%)$; white solid; $[\alpha]_{\mathrm{D}}^{27}+5.82$ (c $\left.0.38, \mathrm{H}_{2} \mathrm{O}\right)$; mp 226$228{ }^{\circ} \mathrm{C}\left\{\right.$ lit. $^{28}[\alpha]_{\mathrm{D}}{ }^{20}+5.90\left(\mathrm{c} 0.38 \mathrm{H}_{2} \mathrm{O}\right)$; $\left.\mathrm{mp} 228-230{ }^{\circ} \mathrm{C}\right\}$.

IR (thin film): 3394, 2926, 1739, 1651, $1454 \mathrm{~cm}^{-1}$.

${ }^{1} \mathrm{H}$ NMR $\left(\mathrm{CD}_{3} \mathrm{OD}, 500 \mathrm{MHz}\right): \delta=7.26-7.18(\mathrm{~m}, 5 \mathrm{H}), 4.38($ br s, $2 \mathrm{H})$, $4.08(\mathrm{~d}, J=5.0 \mathrm{~Hz}, 2 \mathrm{H}), 3.72(\mathrm{~d}, J=10.0 \mathrm{~Hz}, 1 \mathrm{H}), 3.02-3.00(\mathrm{~m}, 1 \mathrm{H})$, 2.83-2.79 (m, $1 \mathrm{H}), 1.65-1.61(\mathrm{~m}, 3 \mathrm{H}), 0.90(\mathrm{~d}, J=5.0 \mathrm{~Hz}, 3 \mathrm{H}), 0.86$ (d, $J=5.0 \mathrm{~Hz}, 3 \mathrm{H})$.

${ }^{13} \mathrm{C}$ NMR $\left(\mathrm{CD}_{3} \mathrm{OD}, 125 \mathrm{MHz}\right): \delta=173.6,173.2,137.0,130.4,130.0$, 128.4, 72.0, 62.5, 56.9, 52.0, 41.0, 34.3, 25.9, 23.2, 21.7, 14.4.

HRMS (ESI-TOF): $m / z[\mathrm{M}+\mathrm{H}]^{+}$calcd for $\mathrm{C}_{16} \mathrm{H}_{25} \mathrm{~N}_{2} \mathrm{O}_{4}$ : 309.1814; found: 309.1812 .

((2S,3R)-3-Amino-2-hydroxy-4-phenylbutanoyl)-L-valyl-L-phenylalanine (2)

Yield: $0.068 \mathrm{~g}(95 \%)$; white solid; $[\alpha]_{\mathrm{D}}{ }^{27}-12.20$ (c 1.02, $\mathrm{H}_{2} \mathrm{O}$ ); mp 187$189^{\circ} \mathrm{C}\left\{\right.$ lit. ${ }^{19}[\alpha]_{\mathrm{D}}{ }^{20}-11.9$ (c $\left.\left.1.00, \mathrm{HOAc}\right) ; 188-191{ }^{\circ} \mathrm{C}\right\}$.

IR (thin film): 2924, 2853, 1732, 1647, $1456 \mathrm{~cm}^{-1}$.

${ }^{1} \mathrm{H}$ NMR (DMSO- $\left.d_{6}, 500 \mathrm{MHz}\right): \delta=8.41(\mathrm{~d}, J=10.0 \mathrm{~Hz}, 1 \mathrm{H}), 8.06$ (br s, $1 \mathrm{H}), 7.81(\mathrm{~d}, J=10.0 \mathrm{~Hz}, 1 \mathrm{H}), 7.34-7.11(\mathrm{~m}, 10 \mathrm{H}), 6.83(\mathrm{br} \mathrm{s}, 1 \mathrm{H})$, 4.43-4.39 (m, $1 \mathrm{H}), 4.19-4.16(\mathrm{~m}, 1 \mathrm{H}), 4.02(\mathrm{~s}, 1 \mathrm{H}), 3.55(\mathrm{~s}, 1 \mathrm{H})$, 3.06-2.90 (m, $4 \mathrm{H}), 2.02-1.98(\mathrm{~m}, 1 \mathrm{H}), 0.84-0.82(\mathrm{~m}, 6 \mathrm{H})$.

${ }^{13} \mathrm{C}$ NMR (DMSO- $\left.d_{6}, 125 \mathrm{MHz}\right): \delta=172.6,170.5,137.5,136.4,129.5$, 129.1, 128.6, 128.1, 126.9, 126.4, 68.2, 57.2, 54.2, 53.5, 36.5, 34.6, $30.7,29.0,19.0,18.0$

HRMS (ESI-TOF): $m / z[\mathrm{M}+\mathrm{H}]^{+}$calcd for $\mathrm{C}_{24} \mathrm{H}_{32} \mathrm{~N}_{3} \mathrm{O}_{5}: 442.2342$; found: 442.2343 .

\section{(3S,4R)-4-Amino-3-hydroxy-5-phenylpentanoic Acid (3)} Yield: $0.032 \mathrm{~g}$ (98\%); clear oil; $[\alpha]_{\mathrm{D}}{ }^{27}-1.50$ (c 0.13, MeOH). IR (thin film): 3405, 2925, 2854, 1737, $1458 \mathrm{~cm}^{-1}$.

${ }^{1} \mathrm{H}$ NMR $\left(\mathrm{D}_{2} \mathrm{O}, 500 \mathrm{MHz}\right): \delta=7.44-7.33(\mathrm{~m}, 5 \mathrm{H}), 4.18-4.14(\mathrm{~m}, 1 \mathrm{H})$, 3.62-3.58 (m, $1 \mathrm{H}), 3.15(\mathrm{dd}, J=15.0,5.0 \mathrm{~Hz}, 1 \mathrm{H}), 2.89$ (dd, $J=15.0$, $10.0 \mathrm{~Hz}, 1 \mathrm{H}), 2.82-2.78(\mathrm{~m}, 1 \mathrm{H}), 2.64(\mathrm{dd}, J=15.0,10.0 \mathrm{~Hz}, 1 \mathrm{H})$.

${ }^{13} \mathrm{C}$ NMR $\left(\mathrm{D}_{2} \mathrm{O}, 125 \mathrm{MHz}\right): \delta=174.8,135.1,129.4,129.2,127.7,66.8$, 56.6, 38.7, 35.3. 
HRMS (ESI-TOF): $m / z$ [M + H] $]^{+}$calcd for $\mathrm{C}_{11} \mathrm{H}_{16} \mathrm{NO}_{3}: 210.1130$; found: 210.1129.

\section{Synthesis of Dipeptide Boc-Val-Phe-OMe}

Boc-Val-OH (0.20 g, $0.92 \mathrm{mmol}$ ) was dissolved in anhydrous DCM (10 $\mathrm{mL}$ ) and the solution was cooled in an ice bath followed by addition of EDC. $\mathrm{HCl}(0.35 \mathrm{~g}, 1.84 \mathrm{mmol})$ and HOBt $(0.24 \mathrm{~g}, 1.84 \mathrm{mmol})$ and the mixture was stirred for $20 \mathrm{~min}$. $\mathrm{HCl} \cdot \mathrm{H}_{2} \mathrm{~N}-\mathrm{Phe}-\mathrm{OMe}(0.19 \mathrm{~g}, 0.92$ mmol) was added to the reaction mixture followed by DIPEA $(0.35$ $\mathrm{mL}, 2.03 \mathrm{mmol}$ ) and the mixture was stirred at r.t. for $6 \mathrm{~h}$. On complete disappearance of starting material, the organic layer was washed with aqueous citric acid $(3 \times 15 \mathrm{~mL})$ and $2 \mathrm{M}$ aqueous $\mathrm{NaHCO}_{3}$ $(3 \times 15 \mathrm{~mL})$. The organic layers were combined, dried over $\mathrm{Na}_{2} \mathrm{SO}_{4}$, filtered, concentrated under vacuum, and purified by column chromatography.

\section{Methyl (tert-Butoxycarbonyl)-L-valyl-L-phenylalaninate (12)}

Column chromatography (petroleum ether/EtOAc, 70:30).

Yield: $0.31 \mathrm{~g}$ (90\%); white solid; $[\alpha]_{\mathrm{D}}{ }^{27}+30.38$ (c $\left.0.88, \mathrm{CHCl}_{3}\right)$; mp 101$103^{\circ} \mathrm{C}$.

IR (thin film): 3361, 3287, 3094, 2958, 2929, 2871, 1746, 1691, 1656, $1567,1514 \mathrm{~cm}^{-1}$.

${ }^{1} \mathrm{H} \mathrm{NMR}\left(\mathrm{CDCl}_{3}, 500 \mathrm{MHz}\right): \delta=7.28-7.20(\mathrm{~m}, 3 \mathrm{H}), 7.09(\mathrm{~d}, J=7.3 \mathrm{~Hz}$, $2 \mathrm{H}$ ), 6.40 (br s, $1 \mathrm{H}), 5.05$ (br s, $1 \mathrm{H}), 4.85(\mathrm{dd}, J=15.0,5.0 \mathrm{~Hz}, 1 \mathrm{H})$, $3.90(\mathrm{~m}, 1 \mathrm{H}), 3.68$ (d, $J=1.4 \mathrm{~Hz}, 3 \mathrm{H}), 3.10-3.07(\mathrm{~m}, 2 \mathrm{H}), 2.08-2.04$ $(\mathrm{m}, 1 \mathrm{H}), 1.43(\mathrm{~s}, 9 \mathrm{H}), 0.90(\mathrm{~d}, J=5.0 \mathrm{~Hz}, 3 \mathrm{H}), 0.84(\mathrm{~d}, J=5.0 \mathrm{~Hz}, 3 \mathrm{H})$. ${ }^{13} \mathrm{C}$ NMR $\left(\mathrm{CDCl}_{3}, 125 \mathrm{MHz}\right): \delta=171.8,171.3,155.8,135.8,129.3$, 128.7, 127.2, 79.9, 59.9, 53.23, 52.3, 38.0, 30.9, 28.4, 19.2, 17.7 .

HRMS (ESI-TOF): $m / z[\mathrm{M}+\mathrm{Na}]^{+}$calcd for $\mathrm{C}_{20} \mathrm{H}_{30} \mathrm{~N}_{2} \mathrm{NaO}_{5}: 401.2052$; found: 401.2052 .

\section{Synthesis of Tripeptide 13}

TFA $(1.00 \mathrm{~mL})$ was added to a stirred solution of Boc-Val-Phe-OMe $(0.22 \mathrm{~g}, 0.58 \mathrm{mmol})$ in anhydrous DCM $(4 \mathrm{~mL})$ at $0{ }^{\circ} \mathrm{C}$ and the mixture was stirred for $30 \mathrm{~min}$. After completion of the reaction as observed in TLC, the solvent was removed under vacuum with addition of DCM ( 5 $\mathrm{mL}, 3$ to 4 times). The residue $(0.20 \mathrm{~g}, 0.58 \mathrm{mmol})$ was dissolved in anhydrous DCM $(10 \mathrm{~mL})$ in an ice bath, followed by addition of EDC. $\mathrm{HCl}(0.22 \mathrm{~g}, 1.18 \mathrm{mmol})$ and HOBt $(0.15 \mathrm{~g}, 1.18 \mathrm{mmol})$ and stirred for $20 \mathrm{~min}$. Boc deprotected dipeptide $12(0.22 \mathrm{~g}, 0.58 \mathrm{mmol})$ was added to the reaction mixture followed by DIPEA $(0.20 \mathrm{~mL}, 1.30$ $\mathrm{mmol}$ ). The reaction mixture was stirred at r.t. for a further $6 \mathrm{~h}$. On complete disappearance of starting material, the organic layer was washed with aqueous citric acid $(3 \times 15 \mathrm{~mL})$ and $2 \mathrm{M}$ aqueous $\mathrm{NaHCO}_{3}$ $(3 \times 15 \mathrm{~mL})$. The organic layers were combined, dried over $\mathrm{Na}_{2} \mathrm{SO}_{4}$, filtered, concentrated, and purified by column chromatography.

\section{Methyl ((2S,3R)-3-((tert-Butoxycarbonyl)amino)-2-(methoxy- methoxy)-4-phenylbutanoyl)-L-valyl-L-phenylalaninate (13) Column chromatography (petroleum ether/EtOAc, 70:30).}

Yield: $0.24 \mathrm{~g}(70 \%)$; white solid; $[\alpha]_{\mathrm{D}}^{27}+18.26$ (c $0.56, \mathrm{CHCl}_{3}$ ); mp $135-137{ }^{\circ} \mathrm{C}$.

IR (thin film): 3295, 3064, 3029, 2923, 2854, 1747, 1692, 1650, 1531, $1455 \mathrm{~cm}^{-1}$.

${ }^{1} \mathrm{H} \mathrm{NMR}\left(\mathrm{CDCl}_{3}, 500 \mathrm{MHz}\right): \delta=7.22-7.13(\mathrm{~m}, 9 \mathrm{H}), 7.05-7.01(\mathrm{~m}, 3 \mathrm{H})$, $6.28(\mathrm{~d}, J=5.1 \mathrm{~Hz}, 1 \mathrm{H}), 5.10(\mathrm{~d}, J=9.9 \mathrm{~Hz}, 1 \mathrm{H}), 4.76(\mathrm{dd}, J=10.0$, $5.0 \mathrm{~Hz}, 1 \mathrm{H}), 4.64-4.60(\mathrm{~m}, 2 \mathrm{H}), 4.21(\mathrm{dd}, J=10.0,5.0 \mathrm{~Hz}, 2 \mathrm{H}), 4.00(\mathrm{~d}$, $J=2.0 \mathrm{~Hz}, 1 \mathrm{H}), 3.65(\mathrm{~s}, 3 \mathrm{H}), 3.34(\mathrm{~s}, 3 \mathrm{H}), 3.06(\mathrm{dd}, J=10.0,5.0 \mathrm{~Hz}$,
$1 \mathrm{H}), 2.97(\mathrm{dd}, J=10.0,5.0 \mathrm{~Hz}, 1 \mathrm{H}), 2.82(\mathrm{dd}, J=10.0,5.0 \mathrm{~Hz}, 1 \mathrm{H}), 2.63$ (dd, $J=13.6,10.0 \mathrm{~Hz}, 1 \mathrm{H}), 2.06-2.04(\mathrm{~m}, 1 \mathrm{H}), 1.24(\mathrm{~s}, 9 \mathrm{H}), 0.86(\mathrm{~d}, J=$ $6.6 \mathrm{~Hz}, 3 \mathrm{H}), 0.81(\mathrm{~d}, J=6.5 \mathrm{~Hz}, 3 \mathrm{H})$.

${ }^{13} \mathrm{C}$ NMR $\left(\mathrm{CDCl}_{3}, 125 \mathrm{MHz}\right): \delta=171.7,170.5,154.9,137.8,135.7$, 129.4, 129.3, 128.7, 128.5, 127.3, 126.5, 97.4, 79.3, 78.8, 58.0, 56.8, 53.5, 53.3, 52.4, 38.1, 37.9, 31.1, 29.8, 28.4, 19.3, 17.8 .

HRMS (ESI-TOF): $m / z[\mathrm{M}+\mathrm{Na}]^{+}$calcd for $\mathrm{C}_{32} \mathrm{H}_{46} \mathrm{~N}_{3} \mathrm{O}_{8}: 600.3285$; found: 600.3282 .

\section{Procedure for Hydrolysis of $\mathbf{1 3}$}

$\mathrm{LiOH}(0.030 \mathrm{~g}, 0.48 \mathrm{mmol})$ was added to a stirred solution of $\mathbf{1 3}(0.24$ g, $0.40 \mathrm{mmol})$ in $\mathrm{MeOH} / \mathrm{H}_{2} \mathrm{O}(4: 1,10 \mathrm{~mL})$ at $0{ }^{\circ} \mathrm{C}$ and the reaction mixture was stirred for $1 \mathrm{~h}$ at the same temperature. After the disappearance of starting material as observed in TLC, the reaction was quenched with saturated aqueous $\mathrm{KHSO}_{4}(10 \mathrm{~mL})$ and the free acid was extracted with EtOAc $(2 \times 40 \mathrm{~mL})$. The organic layers were combined, dried over $\mathrm{Na}_{2} \mathrm{SO}_{4}$, filtered, concentrated under vacuum, and purified by column chromatography.

((2S,3R)-3-((tert-Butoxycarbonyl)amino)-2-(methoxymethoxy)-4phenylbutanoyl)-L-valyl-L-phenylalanine (14)

Column chromatography (DCM/MeOH, 95:5).

Yield: $0.22 \mathrm{~g}$ (94\%); white solid; $[\alpha]_{\mathrm{D}}{ }^{27}+15.69$ (c 0.86, $\mathrm{CHCl}_{3}$ ); mp $109-110{ }^{\circ} \mathrm{C}$.

IR (thin film): 3312, 3064, 3029, 2962, 2925, 2854, 1716, 1650, 1524 $\mathrm{cm}^{-1}$.

${ }^{1} \mathrm{H}$ NMR $\left(\mathrm{CDCl}_{3}, 500 \mathrm{MHz}\right): \delta=7.47(\mathrm{br} \mathrm{s}, 1 \mathrm{H}), 7.27-7.13(\mathrm{~m}, 10 \mathrm{H})$, 6.07 (br s, $1 \mathrm{H}), 5.07$ (d, J = 5.0 Hz, $1 \mathrm{H}), 4.81$ (br s, $1 \mathrm{H}), 4.61-4.59(\mathrm{~m}$, $2 \mathrm{H}$ ), 4.34-4.07 (m, $3 \mathrm{H}), 3.39$ (s, $3 \mathrm{H}), 2.90-2.71(\mathrm{~m}, 3 \mathrm{H}), 2.04$ (br s, $1 \mathrm{H}), 1.76-1.61(\mathrm{~m}, 1 \mathrm{H}), 1.25(\mathrm{~s}, 9 \mathrm{H}), 0.89-0.84(\mathrm{~m}, 6 \mathrm{H})$.

${ }^{13} \mathrm{C}$ NMR $\left(\mathrm{CDCl}_{3}, 125 \mathrm{MHz}\right): \delta=175.7,174.2,173.7,171.0,170.7$, 156.6, 155.1, 137.9, 137.6, 136.1, 129.6, 128.6, 127.1, 126.7, 115.5, 97.3, 96.9, 81.1, 79.6, 78.5, 58.4, 58.2, 56.9, 55.7, 53.7, 53.3, 39.7, 38.5, $37.8,31.8,31.1,29.8,28.3,27.9,20.8,19.4,18.3$.

HRMS (ESI-TOF): $m / z[\mathrm{M}+\mathrm{H}]^{+}$calcd for $\mathrm{C}_{31} \mathrm{H}_{44} \mathrm{~N}_{3} \mathrm{O}_{8}: 586.3128$; found: 586.3121.

\section{Asymmetric $\alpha$-Hydroxylation of Aldehyde 4}

L-Proline $(0.13 \mathrm{~g}, 1.14 \mathrm{mmol}, 30 \mathrm{~mol} \%)$ and nitrosobenzene $(0.44 \mathrm{~g}$, $4.18 \mathrm{mmol})$ were added to a stirred solution of $4(1.00 \mathrm{~g}, 3.80 \mathrm{mmol})$ in anhydrous DMSO $(10 \mathrm{~mL})$ at $15^{\circ} \mathrm{C}$ and the mixture was stirred for 3 $\mathrm{h}$ at the same temperature. After $3 \mathrm{~h}$ the reaction was cooled to $0{ }^{\circ} \mathrm{C}$ and phosphorane $\mathrm{Ph}_{3} \mathrm{P}=\mathrm{CHCO}_{2} \mathrm{Et}(2.65 \mathrm{~g}, 7.60 \mathrm{mmol})$ in $\mathrm{DCM}(10 \mathrm{~mL})$ was added and the reaction mixture was stirred for a further $2 \mathrm{~h}$ at $0{ }^{\circ} \mathrm{C}$. On complete disappearance of starting material, the reaction was quenched with saturated aqueous $\mathrm{NH}_{4} \mathrm{Cl}(30 \mathrm{~mL})$ and the mixture was extracted with DCM $(2 \times 30 \mathrm{~mL})$. The combined organic phases were washed with brine ( $30 \mathrm{~mL}$ ), dried over $\mathrm{Na}_{2} \mathrm{SO}_{4}$, filtered, and concentrated under vacuum. The crude aminohydroxylated product was taken as such to the next step, leading to the cleavage of $\mathrm{O}-\mathrm{N}$ bond.

$\mathrm{Cu}(\mathrm{OAc})_{2}(0.17 \mathrm{~g}, 0.96 \mathrm{mmol})$ was added to a stirred solution of the above product $(1.43 \mathrm{~g}, 3.24 \mathrm{mmol})$ in $\mathrm{EtOH}(10 \mathrm{~mL})$ and the mixture stirred at r.t. for $6 \mathrm{~h}$. On complete disappearance of starting material, the reaction was quenched with saturated aqueous $\mathrm{NH}_{4} \mathrm{Cl}(20 \mathrm{~mL})$ and the mixture was extracted with DCM $(2 \times 20 \mathrm{~mL})$. The combined organic phases were washed with brine $(30 \mathrm{~mL})$, dried over $\mathrm{Na}_{2} \mathrm{SO}_{4}$, filtered, concentrated under vacuum, and purified by column chromatography. 
Ethyl (4S,5R,E)-5-((tert-Butoxycarbonyl)amino)-4-hydroxy-6phenylhex-2-enoate (15)

Column chromatography (petroleum ether/EtOAc, 80:20).

Yield: $0.80 \mathrm{~g}$ (70\%); clear oil; $[\alpha]_{\mathrm{D}}{ }^{27}-3.91$ (c $0.23, \mathrm{CHCl}_{3}$ ).

IR (thin film): 3355, 2926, 1729, 1683, $1524 \mathrm{~cm}^{-1}$.

${ }^{1} \mathrm{H} \mathrm{NMR}\left(\mathrm{CDCl}_{3}, 500 \mathrm{MHz}\right): \delta=7.31-7.17(\mathrm{~m}, 5 \mathrm{H}), 6.98(\mathrm{dd}, J=15.0$, $5.0 \mathrm{~Hz}, 1 \mathrm{H}), 6.15(\mathrm{~d}, J=15.0,5.0 \mathrm{~Hz}, 1 \mathrm{H}), 4.62(\mathrm{~d}, J=10.0 \mathrm{~Hz}, 1 \mathrm{H})$, 4.43 (br s, $1 \mathrm{H}), 4.21$ (q, J = 5.0 Hz, $2 \mathrm{H}), 4.02(\mathrm{~s}, 1 \mathrm{H}), 3.81(\mathrm{~s}, 1 \mathrm{H})$, 2.84-2.77 (m, $2 \mathrm{H}), 1.36(\mathrm{~s}, 9 \mathrm{H}), 1.29$ (t, J= 5.0 Hz, $3 \mathrm{H})$.

${ }^{13} \mathrm{C}$ NMR $\left(\mathrm{CDCl}_{3}, 125 \mathrm{MHz}\right): \delta=166.4,157.0,146.0,137.4,129.2$, $128.8,126.9,122.8,80.5,73.6,60.6,57.0,36.2,29.8,28.3,14.3$.

HRMS (ESI-TOF): $m / z[\mathrm{M}+\mathrm{Na}]^{+}$calcd for $\mathrm{C}_{19} \mathrm{H}_{27} \mathrm{NNaO}_{5}: 372.1787$; found: 372.1772 .

\section{Procedure for Oxazolidine Protection of 15}

A catalytic amount of $p$ - $\mathrm{TsOH}(0.09 \mathrm{~g}, 0.57 \mathrm{mmol})$ and dimethoxypropane $(1.11 \mathrm{~mL}, 8.59 \mathrm{mmol})$ were added to a stirred solution of $\mathbf{1 5}$ $(1.00 \mathrm{~g}, 2.86 \mathrm{mmol})$ in anhydrous DCM $(20 \mathrm{~mL})$ and the mixture was stirred at r.t. for $2 \mathrm{~h}$. On complete disappearance of starting material, the reaction was quenched with saturated aqueous $\mathrm{NaHCO}_{3}(20 \mathrm{~mL})$ and the crude product was extracted with DCM $(2 \times 30 \mathrm{~mL})$. The combined organic phases were dried over $\mathrm{Na}_{2} \mathrm{SO}_{4}$, filtered, concentrated under vacuum, and purified by column chromatography.

tert-Butyl (4R,5S)-4-Benzyl-5-((E)-3-ethoxy-3-oxoprop-1-en-1-yl)2,2-dimethyloxazolidine-3-carboxylate (16)

Column chromatography (petroleum ether/EtOAc, 85:15).

Yield: $0.95 \mathrm{~g}$ (85\%); clear oil; $[\alpha]_{\mathrm{D}}{ }^{27}-13.77$ (c $0.80, \mathrm{CHCl}_{3}$ ).

IR (thin film): 2978, 2930, 1723, 1701, $1604 \mathrm{~cm}^{-1}$.

${ }^{1} \mathrm{H} \mathrm{NMR}\left(\mathrm{CDCl}_{3}, 400 \mathrm{MHz}\right): \delta=7.29-7.10(\mathrm{~m}, 5 \mathrm{H}), 6.61-6.54(\mathrm{~m}, 1 \mathrm{H})$, 6.16-6.12 (m, $1 \mathrm{H}), 4.69$ (br s, $1 \mathrm{H}), 4.47-4.24(\mathrm{~m}, 1 \mathrm{H}), 4.19-4.10(\mathrm{~m}$, $2 \mathrm{H}), 3.22$ (dd $J=12.0,4.0 \mathrm{~Hz}, 1 \mathrm{H}), 2.91-2.79(\mathrm{~m}, 1 \mathrm{H}), 2.71-2.66(\mathrm{~m}$, $1 \mathrm{H}), 1.56(\mathrm{~s}, 3 \mathrm{H}), 1.52(\mathrm{~s}, 3 \mathrm{H}), 1.46(\mathrm{~s}, 3 \mathrm{H}), 1.37$ (s, $3 \mathrm{H}), 1.28-1.22$ $(\mathrm{m}, 6 \mathrm{H})$.

${ }^{13} \mathrm{C}$ NMR $\left(\mathrm{CDCl}_{3}, 125 \mathrm{MHz}\right): \delta=165.8,151.9,151.5,141.7,141.6$, 138.2, 137.1, 130.0, 129.9, 128.4, 128.2, 126.3, 126.2, 122.6, 93.7, 93.0, $80.4,80.1,75.7,75.4,63.2,61.7,61.3,60.6,60.5,37.4,36.6,28.6,28.5$, 28.0, 27.4, 27.0, 25.2, 24.0, 14.3, 14.2.

HRMS (ESI-TOF): $m / z[\mathrm{M}+\mathrm{Na}]^{+}$calcd for $\mathrm{C}_{22} \mathrm{H}_{31} \mathrm{NNaO}_{5}: 412.2100$; found: 412.2105 .

\section{$\mathbf{L i B H}_{\mathbf{4}}$ Reduction of 16}

$\mathrm{LiBH}_{4}(0.17 \mathrm{~g}, 7.70 \mathrm{mmol})$ was added to a stirred solution of $\mathbf{1 6}$ (1.00 $\mathrm{g}, 2.57 \mathrm{mmol})$ in anhydrous THF $(20 \mathrm{~mL})$ at $0{ }^{\circ} \mathrm{C}$ and the mixture was stirred at r.t. for $8 \mathrm{~h}$. On complete disappearance of starting material, the reaction was quenched with saturated aqueous $\mathrm{NaHCO}_{3}(20 \mathrm{~mL})$. The crude product was extracted with EtOAc $(2 \times 30 \mathrm{~mL})$ and the combined organic phases were dried over $\mathrm{Na}_{2} \mathrm{SO}_{4}$, filtered, concentrated under vacuum, and purified by column chromatography.

tert-Butyl (4R,5S)-4-Benzyl-5-(3-hydroxypropyl)-2,2-dimethyloxazolidine-3-carboxylate (17)

Column chromatography (petroleum ether/EtOAc, 70:30).

Yield: $0.72 \mathrm{~g}$ (80\%); clear oil; $[\alpha]_{\mathrm{D}}{ }^{27}+21.04$ (c $0.51, \mathrm{CHCl}_{3}$ ).

IR (thin film): 3445, 3062, 3027, 2928, 2856, 1696, $1604 \mathrm{~cm}^{-1}$.
${ }^{1} \mathrm{H} \mathrm{NMR}\left(\mathrm{CDCl}_{3}, 400 \mathrm{MHz}\right): \delta=7.29-7.14(\mathrm{~m}, 5 \mathrm{H}), 4.26-4.11(\mathrm{~m}, 1 \mathrm{H})$, 4.05-3.86 (m, $1 \mathrm{H}), 3.52-3.50(\mathrm{~m}, 2 \mathrm{H}), 3.22-3.18$ (dd, $J=12.0,4.0 \mathrm{~Hz}$, $1 \mathrm{H}), 2.92-2.81(\mathrm{~m}, 2 \mathrm{H}), 1.66-1.49(\mathrm{~m}, 9 \mathrm{H}), 1.43(\mathrm{~s}, 4 \mathrm{H}), 1.32(\mathrm{~s}, 4 \mathrm{H})$, $1.24(\mathrm{~s}, 2 \mathrm{H})$.

${ }^{13} \mathrm{C}$ NMR $\left(\mathrm{CDCl}_{3}, 100 \mathrm{MHz}\right): \delta=152.0,151.7,139.1,139.1,129.5$, 129.3, 128.5, 128.3, 126.2, 126.1, 93.0, 92.4, 80.1, 79.7, 62.4, 60.9, $60.8,36.5,35.9,29.9,29.8,28.5,28.4,28.1,27.5,26.2,25.0,23.8$.

HRMS (ESI-TOF): $m / z$ [M $+\mathrm{Na}]^{+}$calcd for $\mathrm{C}_{20} \mathrm{H}_{31} \mathrm{NNaO}_{4}: 372.2151$; found: 372.2151 .

\section{Oxidation of Primary Alcohols}

IBX $(0.69 \mathrm{~g}, 2.47 \mathrm{mmol})$ was added to a solution of $\mathbf{1 7}(0.72 \mathrm{~g}, 2.06$ $\mathrm{mmol})$ in DMSO $(10 \mathrm{~mL})$ at r.t. and the mixture was stirred for $3 \mathrm{~h}$. On complete disappearance of starting material, the reaction was quenched with saturated aqueous $\mathrm{NaHCO}_{3}(50 \mathrm{~mL})$ and the mixture was extracted with EtOAc $(2 \times 30 \mathrm{~mL})$. The combined organic phases washed with brine $(30 \mathrm{~mL})$ and dried over $\mathrm{Na}_{2} \mathrm{SO}_{4}$, filtered, concentrated under reduced pressure, and purified by column chromatography.

tert-Butyl (4R,5S)-4-Benzyl-2,2-dimethyl-5-(3-oxopropyl)oxazolidine-3-carboxylate (18)

Column chromatography (petroleum ether/EtOAc, 80:20).

Yield: $0.63 \mathrm{~g}(88 \%)$; clear oil; $[\alpha]_{\mathrm{D}}{ }^{27}+15.25$ (c $0.72, \mathrm{CHCl}_{3}$ ).

IR (thin film): 2927, 2854, 2719, 1727, 1696, $1604 \mathrm{~cm}^{-1}$.

${ }^{1} \mathrm{H} \mathrm{NMR}\left(\mathrm{CDCl}_{3}, 400 \mathrm{MHz}\right): \delta=9.60,9.57(\mathrm{~s}, 1 \mathrm{H}), 7.30-7.15(\mathrm{~m}, 5 \mathrm{H})$, 4.25-4.11 (m, $1 \mathrm{H}), 3.99-3.96$ (m, $1 \mathrm{H}), 2.96-2.79(\mathrm{~m}, 2 \mathrm{H}), 2.44-2.29$ (m, $1 \mathrm{H}), 2.23-2.08(\mathrm{~m}, 1 \mathrm{H}), 1.95-1.79(\mathrm{~m}, 1 \mathrm{H}), 1.69-1.63(\mathrm{~m}, 3 \mathrm{H})$, 1.54-1.50 (m, $4 \mathrm{H}), 1.46-1.44$ (m, $5 \mathrm{H}), 1.35$ (s, $4 \mathrm{H})$.

${ }^{13} \mathrm{C}$ NMR $\left(\mathrm{CDCl}_{3}, 100 \mathrm{MHz}\right): \delta=201.3,152.0,151.6,138.9,129.4$, 129.3, 128.6, 128.4, 126.3, 126.2, 92.9, 92.4, 80.1, 79.8, 76.4, 76.2, 60.8, 60.6, 40.7, 36.5, 35.9, 29.8, 28.5, 28.4, 28.1, 27.4, 25.0, 23.8, 22.1. HRMS (ESI-TOF): $m / z[\mathrm{M}+\mathrm{Na}]^{+}$calcd for $\mathrm{C}_{20} \mathrm{H}_{29} \mathrm{NNaO}_{4}: 370.1994$; found: 370.1992 .

\section{Synthesis of 20 from Diol 19}

$\mathrm{NaIO}_{4}(0.56 \mathrm{~g}, 2.62 \mathrm{mmol})$ was added to a stirred solution of diol $\mathbf{1 9}$ $(0.48 \mathrm{~g}, 1.31 \mathrm{mmol})$ in $\mathrm{DCM} / \mathrm{MeOH}(1: 1,10 \mathrm{~mL})$ and the mixture was stirred at r.t. for $4 \mathrm{~h}$. On complete disappearance of starting material, the reaction mixture was filtered and washed with brine $(20 \mathrm{~mL})$. The crude product was extracted with EtOAc $(2 \times 30 \mathrm{~mL})$ and dried over $\mathrm{Na}_{2} \mathrm{SO}_{4}$, filtered, concentrated under reduced pressure, and purified by column chromatography.

\section{tert-Butyl (4R,5S)-4-Benzyl-2,2-dimethyl-5-(2-oxoethyl)oxazoli-} dine-3-carboxylate (20)

Column chromatography (petroleum ether/EtOAc, 80:20).

Yield: $0.38 \mathrm{~g}$ (85\%); clear oil; $[\alpha]_{\mathrm{D}}{ }^{27}-2.82$ (c $0.49, \mathrm{CHCl}_{3}$ ).

IR (thin film): 3439, 2975, 2931, 1728, 1697, 1495, $1455 \mathrm{~cm}^{-1}$.

${ }^{1} \mathrm{H} \mathrm{NMR}\left(\mathrm{CDCl}_{3}, 500 \mathrm{MHz}\right): \delta=9.55(\mathrm{~s}, 1 \mathrm{H}), 7.30-7.19(\mathrm{~m}, 5 \mathrm{H}), 4.53-$ $4.40(\mathrm{~m}, 1 \mathrm{H}), 3.84(\mathrm{br} \mathrm{s}, 1 \mathrm{H}), 3.32(\mathrm{~d}, J=5.0 \mathrm{~Hz}, 1 \mathrm{H}), 2.76-2.71(\mathrm{~m}$, $1 \mathrm{H}), 2.52-2.46(\mathrm{~m}, 1 \mathrm{H}), 2.21-2.17(\mathrm{~m}, 1 \mathrm{H}), 1.39(\mathrm{~s}, 15 \mathrm{H})$.

${ }^{13} \mathrm{C}$ NMR $\left(\mathrm{CDCl}_{3}, 100 \mathrm{MHz}\right): \delta=200.0,152.2,151.7,138.2,137.3$, 129.7, 129.4, 128.7, 126.9, 95.1, 94.4, 80.4, 74.3, 73.5, 63.3, 48.7, 48.2, 43.7, 39.8, 37.7, 29.8, 28.6, 26.9.

HRMS (ESI-TOF): $m / z[\mathrm{M}+\mathrm{Na}]^{+}$calcd for $\mathrm{C}_{19} \mathrm{H}_{27} \mathrm{NNaO}_{4}: 356.1838$; found: 356.1841 . 


\section{Synthesis of 21 from 20}

Pyridinium dichromate $(0.45 \mathrm{~g}, 1.20 \mathrm{mmol})$ was added to a stirred solution of $20(0.10 \mathrm{~g}, 0.30 \mathrm{mmol})$ in DMF $(10 \mathrm{~mL})$ and stirring was continued at r.t. for $8 \mathrm{~h}$. On complete disappearance of the starting material, the reaction was quenched with water $(100 \mathrm{~mL})$, the crude product was extracted with $\mathrm{Et}_{2} \mathrm{O}(2 \times 40 \mathrm{~mL})$ and the combined organic phases were further extracted with saturated aqueous $\mathrm{NaHCO}_{3}$ $(2 \times 30 \mathrm{~mL})$. The aqueous extracts containing the carboxylate salt were combined and acidified with saturated aqueous $\mathrm{KHSO}_{4}(2 \times 40$ $\mathrm{mL})$ and extracted with $\mathrm{Et}_{2} \mathrm{O}(2 \times 50 \mathrm{~mL})$. The combined organic phases were dried over $\mathrm{Na}_{2} \mathrm{SO}_{4}$, filtered, concentrated under reduced pressure, and purified by column chromatography.

2-((4R,5S)-4-Benzyl-3-(tert-butoxycarbonyl)-2,2-dimethyloxazolidin-5-yl)acetic Acid (21)

Column chromatography (DCM/MeOH, 95:05).

Yleld: $0.07 \mathrm{~g}$ (67\%); clear oil; $[\alpha]_{\mathrm{D}}{ }^{27}-5.77$ (c $0.48, \mathrm{CHCl}_{3}$ ).

IR (thin film): 3478, 2976, 2927, 2854, 1698, 1495, $1455 \mathrm{~cm}^{-1}$.

${ }^{1} \mathrm{H} \mathrm{NMR}\left(\mathrm{CDCl}_{3}, 500 \mathrm{MHz}\right): \delta=7.29-7.25(\mathrm{~m}, 2 \mathrm{H}), 7.21-7.20(\mathrm{~m}, 3 \mathrm{H})$, 4.46-4.34 (m, $1 \mathrm{H}), 3.89-3.83(\mathrm{~m}, 1 \mathrm{H}), 3.26$ (br s, $1 \mathrm{H}), 2.88-2.66(\mathrm{~m}$, $1 \mathrm{H}), 2.49-2.44$ (m, $1 \mathrm{H}), 2.23-2.19(\mathrm{~m}, 1 \mathrm{H}), 1.37$ (s, $15 \mathrm{H})$.

${ }^{13} \mathrm{C} \mathrm{NMR}\left(\mathrm{CDCl}_{3}, 100 \mathrm{MHz}\right): \delta=175.6,152.3,138.2,137.5,129.4$, 128.7, 126.8, 95.1, 94.5, 80.5, 75.7, 75.0, 72.8, 63.4, 59.9, 40.0, 38.0, 28.7, 28.5, 27.3.

HRMS (ESI-TOF): $m / z[\mathrm{M}+\mathrm{Na}]^{+}$calcd for $\mathrm{C}_{19} \mathrm{H}_{27} \mathrm{NNaO}_{5}: 372.1787$; found: 372.1789 .

\section{Synthesis of Aldehyde 4}

To a stirred solution of methoxymethyltriphenyl-phosphonium chloride $(2.05 \mathrm{~g}, 6.02 \mathrm{mmol})$ and $t$-BuOK $(0.58 \mathrm{~g}, 5.21 \mathrm{mmol})$ in anhydrous THF (10 mL), HN-Boc-D-phenyl-alaninal ( $1.00 \mathrm{~g}, 4.01 \mathrm{mmol})$ in anhydrous THF $(10 \mathrm{~mL})$ was added slowly at $-10{ }^{\circ} \mathrm{C}$ and the mixture was stirred vigorously for $2 \mathrm{~h}$ at the same temperature. On complete disappearance of starting material, the reaction was quenched with saturated aqueous $\mathrm{NH}_{4} \mathrm{Cl}(30 \mathrm{~mL})$ and the mixture was extracted with EtOAc $(2 \times 30 \mathrm{~mL})$. The combined organic phases were dried over $\mathrm{Na}_{2} \mathrm{SO}_{4}$, filtered, concentrated under vacuum, and purified by column chromatography using 90:10 petroleum ether/EtOAc as eluent.

$\mathrm{HCl}(2 \mathrm{M}, 5 \mathrm{~mL})$ was added to the above compound (1.02 g, 3.68 $\mathrm{mmol})$ in THF ( $5 \mathrm{~mL})$ at $0{ }^{\circ} \mathrm{C}$ and the mixture was stirred vigorously at $0{ }^{\circ} \mathrm{C}$ for $1 \mathrm{~h}$. On complete disappearance of starting material, the reaction was quenched with saturated aqueous $\mathrm{NaHCO}_{3}(10 \mathrm{~mL})$, and the mixture was extracted with EtOAc $(2 \times 20 \mathrm{~mL})$ and the combined organic phases containing crude product were dried over $\mathrm{Na}_{2} \mathrm{SO}_{4}$, filtered, concentrated, and purified by column chromatography.

\section{tert-Butyl (R)-(4-0xo-1-phenylbutan-2-yl)carbamate (4)}

Column chromatography (petroleum ether/EtOAc, 80:20).

Yield: $0.85 \mathrm{~g}$ (81\%); clear oil; $[\alpha]_{\mathrm{D}}^{27}+12.04$ (c $0.51, \mathrm{CHCl}_{3}$ ).

IR (thin film): 2928, 2856, 2718, 1727, 1696, $1604 \mathrm{~cm}^{-1}$.

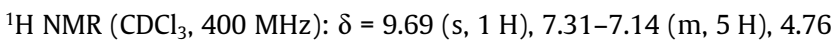
(br s, 1 H), 4.25 (br s, 1 H), 2.95-2.61 (m, 2 H), 2.58-2.48 (m, 2 H), 1.39 (s, $9 \mathrm{H})$.

${ }^{13} \mathrm{C}$ NMR $\left(\mathrm{CDCl}_{3}, 100 \mathrm{MHz}\right): \delta=201.1,155.2,137.5,129.4,128.7$, 126.9, 79.7, 47.7, 47.5, 40.8, 31.3, 29.8, 28.4.

HRMS (ESI-TOF): $m / z[\mathrm{M}+\mathrm{Na}]^{+}$calcd for $\mathrm{C}_{15} \mathrm{H}_{21} \mathrm{NNaO}_{3}: 286.1419$; found: 286.1421 .

\section{Funding Information}

The author thanks CSIR for a Senior Research Fellowship

\section{Acknowledgment}

The author thanks his Ph.D Thesis supervisor Dr. Ramesh Ramapanicker for allowing him to work in his laboratory and for providing assistance, IIT Kanpur for providing instrumental facilities.

\section{Supporting Information}

Supporting information for this article is available online at https://doi.org/10.1055/s-0039-1690223.

\section{References and Notes}

(1) Umezawa, H.; Aoyagi, T.; Suda, H.; Hamada, M.; Takeuchi, T. J. Antibiot. 1976, 29, 97.

(2) Nishino, N.; Powers, J. C. Biochemistry 1979, 18, 4340.

(3) Suda, H.; Takita, T.; Aoyagi, T.; Umezawa, H.J. Antibiot. 1976, 29, 100.

(4) Nakamura, H.; Suda, H.; Takita, T.; Aoyagi, T.; Umezawa, H.; Iitaka, Y. J. Antibiot. 1976, 29, 102.

(5) Pulido-Cejudo, G.; Conway, B.; Proulx, P.; Brown, R.; Izaguirre, C. A. Antiviral Res. 1997, 36, 167.

(6) Bourinbaiar, A. S.; Lee-Huang, S.; Krasinski, K.; Borkowsky, W. Biomed. Pharmacother. 1994, 48, 55.

(7) Inoi, K.; Goto, S.; Nomura, S.; Isobe, K.; Nawa, A.; Okamoto, T.; Tomoda, Y. Anticancer Res. 1995, 15, 2081.

(8) Dzoljic, E.; Varagic, V. M. Fundam. Clin. Pharmacol. 1987, 1, 307.

(9) Harbeson, S. L.; Rich, D. H. Biochemistry 1988, 27, 7301.

(10) Nagai, M.; Kojima, F.; Naganawa, H.; Hamada, M.; Aoyagi, T.; Takeuchi, T. J. Antibiot. 1997, 50, 82.

(11) Gogoi, N.; Boruwa, J.; Barua, N. C. Tetrahedron Lett. 2005, 46, 7581.

(12) Jung, D. Y.; Kang, S.; Chang, S.; Kim, Y. H. Synlett 2006, 86.

(13) Gogoi, N.; Borah, J. C.; Boruwa, J.; Barua, N. C. Lett. Org. Chem. 2007, 4, 234.

(14) Feske, B. D. Curr. Org. Chem. 2007, 11, 483; and therein.

(15) (a) George, S.; Suryavanshi, G. S.; Sudalai, A. Tetrahedron Lett. 2008, 49, 6791; and therein. (b) Venkataramasubramanian, V.; Kiran, I. N. C.; Sudalai, A. Synlett 2015, 26, 355.

(16) Velmourougane, G.; Harbut, M. B.; Dalal, S.; McGowan, S.; Oellig, C. A.; Meinhardt, N.; Whisstock, J. C.; Klemba, M.; Greenbaum, D. C. J. Med. Chem. 2011, 54, 1655.

(17) Wasserman, H. H.; Petersen, A. K.; Xia, M. Tetrahedron 2003, 59, 6771.

(18) Lee, B. W.; Lee, J. H.; Jang, K. C.; Kang, J. E.; Kim, J. H.; Park, K.M.; Park, K. H. Tetrahedron Lett. 2003, 44, 5905.

(19) Righi, G.; Achille, C. D.; Pescatore, G.; Bonini, C. Tetrahedron Lett. 2003, 44, 6999.

(20) Feske, B. D.; Stewart, J. D. Tetrahedron: Asymmetry 2005, 16, 3124.

(21) Kudyba, I.; Raczko, J.; Jurczak, J. Tetrahedron Lett. 2003, 44, 8685.

(22) Seo, Y.; Kim, H.; Chae, D. W.; Kim, Y. G. Tetrahedron: Asymmetry 2014, 25, 625 .

(23) Lee, J. H.; Lee, B. W.; Jang, K. C.; Jeong, I.-Y.; Yang, M. S.; Lee, S. G.; Park, K. H. Synthesis 2003, 829.

(24) Semple, J. E.; Owens, T. D.; Nguyen, K.; Levy, O. Org. Lett. 2000, 2,2769 . 
(25) Nemoto, H.; Ma, R.; Suzuki, I.; Shibuya, M. Org. Lett. 2000, 2, 4245.

(26) Kudyba, I.; Raczko, J.; Jurczak, J. J. Org. Chem. 2004, 69, 2844.

(27) (a) Bergmeier, S. C.; Stanchina, D. M. J. Org. Chem. 1999, 64, 2852. (b) Shang, S.; Willems, A. V.; Chauhan, S. S. J. Pept. Sci. 2018, 24, 3067.

(28) Richter, A.; Hedberg, C. Synthesis 2010, 2039.

(29) Lee, J. H.; Kim, J. H.; Lee, B. W.; Seo, W. D.; Yang, M. S.; Park, K. H. Bull. Korean Chem. Soc. 2006, 27, 1211.

(30) (a) see ref 15b (b) Kumar, P.; Dwivedi, N. Acc. Chem. Res. 2013, 46, 289. (c) Zhong, G. Angew. Chem. Int. Ed. 2003, 42, 4247. (d) Mukherjee, S.; Yang, J. W.; Hoffmann, S.; List, B. Chem. Rev. 2007, 107, 5471. (e) Vilaivan, T.; Bhanthumnavin, W. Molecules 2010, 15, 917. (f) Lalwani, K. G.; Sudalai, A. Synlett 2016, 27, 1339. (g) Hayashi, Y.; Yamaguchi, J.; Hibino, K.; Shoji, M. Tetrahedron Lett. 2003, 44, 8293. (h) Mangion, I. K.; MacMillan, D. W. C. J. Am. Chem. Soc. 2005, 127, 3696. (i) Momiyama, N.; Yamamoto, H. J. Am. Chem. Soc. 2003, 125, 6038. (j) Lee, L. G.; Whitesides, G. M. J. Org. Chem. 1986, 51, 25. (k) Brown, S. P.; Brochu, M. P.; Sinz, C. J.; MacMillan, D. W. C. J. Am. Chem. Soc. 2003, 125, 10808.

(31) Janey, J. M. Angew. Chem. Int. Ed. 2005, 44, 4292.

(32) Chacko, S.; Ramapanicker, R. J. Org. Chem. 2015, 80, 4776

(33) Chacko, S.; Kalita, M.; Ramapanicker, R. Tetrahedron: Asymmetry 2015, 26, 623.

(34) Petakamsetty, R.; Jain, V. K.; Majhi, P. K.; Ramapanicker, R. Org. Biomol. Chem. 2015, 13, 8512.

(35) Chacko, S.; Ramapanicker, R. Tetrahedron Lett. 2015, 56, 2023.

(36) Petakamsetty, R.; Das, R. P.; Ramapanicker, R. Tetrahedron 2014, $70,9554$.

(37) Chacko, S.; Ramapanicker, R. Eur. J. Org. Chem. 2012, 7120.

(38) Philip, A. T.; Chacko, S.; Ramapanicker, R. Synthesis 2013, 45, 1997.
(39) Philip, A. T.; Chacko, S.; Ramapanicker, R. J. Pept. Sci. 2015, 21, 887.

(40) Chacko, S.; Ramapanicker, R. ChemistrySelect 2016, 1, 4458.

(41) Petakamsetty, R.; Ansari, A.; Ramapanicker, R. Carbohydr. Res. 2016, 435, 37.

(42) Jain, V. K.; Ramapanicker, R. Tetrahedron 2017, 73, 1568.

(43) For synthesis of aldehyde 4 see: (a) Guduru, S. K. R.; Chamakuri, S.; Raji, I. O.; MacKenzie, K. R.; Santini, C.; Young, D. W. J. Org. Chem. 2018, 83, 11777. (b) Cytlak, T.; Skibinska, M.; Kaczmarek, P.; Kazmierczak, M.; Rapp, M.; Kubicki, M.; Koroniak, H. RSC Adv. 2018, 8, 11957. (c) Shankar, P. S.; Bigotti, S.; Lazzari, P.; Manca, I.; Spiga, M.; Sani, M.; Zanda, M. Tetrahedron Lett. 2013, 54, 6137. (d) Yao, L.; Wen, J.; Liu, S.; Tan, R.; Wood, N. M.; Chen, W.; Zhang, S.; Zhang, X. Chem. Commun. 2016, 52, 2273; See also the Experimental Section 'Procedure for Synthesis of Aldehyde 4'.

(44) Umezawa, H.; Aoyagi, T.; Morishima, H.; Matsuzaki, M.; Hamada, M.; Takeuchi, T. J. Antibiot. 1970, 23, 259.

(45) Moore, M. L.; Bryan, W. M.; Fakhoury, S. A.; Magaard, V. W.; Huffman, W. F.; Dayton, B. D.; Meek, T. D.; Hyland, L.; Dreyer, G. B.; Metcalf, B. W.; Strickler, J. E.; Gorniak, J. G.; Debouck, C. Biochem. Biophys. Res. Commun. 1989, 159, 420.

(46) Liu, J.; Chen, W.; Xu, Y.; Ren, S.; Zhang, W.; Li, Y. Bioorg. Med. Chem. 2015, 23, 1963.

(47) Si, C.-M.; Shao, L.-P.; Mao, Z.-Y.; Zhou, W.; Wei, B.-G. Org. Biomol. Chem. 2017, 15, 649.

(48) Li, X.; Li, Y.-l.; Chen, Y.; Zou, Y.; Zhuo, X.-b.; Wu, Q.-y.; Zhao, Q.j.; Hu, H.-g. RSC Adv. 2015, 5, 94654.

(49) Kondekar, N. B.; Kandula, S. R. V.; Kumar, P. Tetrahedron Lett. 2004, 45, 5477.

(50) Andres, J. M.; Pedrosa, R.; Perez, A.; Encabo, A. P. Tetrahedron 2001, 57, 8521. 